\title{
PRODUCTS OF STOCHASTIC MATRICES AND APPLICATIONS
}

\author{
HARRY COHN \\ Department of Statistics \\ The University of Melbourne \\ Parkville, Victoria 3052 \\ Australia
}

(Received November 20, 1987)

ABSTRACT. This paper deals with aspects of the limit behaviour of products of nonidentical finite or countable stochastic matrices $\left(P_{n}\right)$. Applications are given to nonhomogeneous Markov models as positive chains, some classes of finite chains considered by Doeblin and weakly ergodic chains.

KEY WORDS AND PHRASES. Stochastic matrix, nonhomogeneity, Markov chains, weak ergodicity, tail $\sigma$-field, atomic set. 1980 AMS SUBJECTS CLASSIFICATION CODES. 15A51, 60J10.

\section{INTRODUCTION.}

Let $P_{0}, P_{1}, \ldots$, be a sequence of finite or countable stochastic matrices,
$P_{i, j}^{(n)}$ the $(i, j)$ entry of $P_{n}, P_{m, n}=P_{m} \ldots P_{n}^{(m, n)}$ the $(i, j)$ entry of $P_{m, n}$. In the 'homogeneous' case, when $P=P_{0}=\ldots$, the classical Markov chains theory provides a detailed analysis of $\mathrm{P}^{\mathrm{n}}$ : for ergodic chains $\mathrm{P}^{\mathrm{n}}$ converges as $\mathrm{n} \rightarrow \infty$, whereas otherwise $\mathrm{P}^{\mathrm{nd}+\mathrm{r}}$ converges as $\mathrm{n} \rightarrow \infty$ for some $\mathrm{d}>1$ and $r=1, \ldots, d-1$. It turns out that in the 'nonhomogeneous' case, when $\left(P_{n}\right)$ are nonidentical, $\lim \inf _{n \rightarrow \infty} P_{i, j}^{(m, n)}>0$ and $P_{\ell, j}^{(m, n)}>0$ imply that $\left\{P_{i, j}^{(m, n)} / P(m, n)\right\}$ converges as $n \rightarrow \infty$ in a case that may be thought of as aperiodic whereas otherwise $\left\{P_{i, j}^{(m, n)} / P_{\ell, j}^{(m, n)}: n \geqslant m\right\}$ assume a finite number of 1 imit points. Both $\lim _{n \rightarrow \infty} P_{i, j}^{(m, n)} / P_{\ell, j}^{(m, n)}$ and the limit points of $\left\{P_{i, j}^{(m, n)} / P_{\ell, j}^{(m, n)}: n=m+1, \ldots\right\}$ will be identified in terms of $\alpha_{i}^{(m)}(k) / \alpha_{\ell}^{(m)}(k)$ where $\alpha_{u}^{(m)}(k)=1 i{ }_{n \rightarrow \infty} \sum_{i \in E}(k) p_{n}^{(m, n)}$ for some sequence of sets $\left\{E_{n}^{(k)}\right\}$. Our results may be understood without reference to Markov chains, but the proofs will consider a Markov chain $\left\{x_{n}: n \geqslant m\right\}$ with finite or countable state spaces assuming a strictly positive initial probability vector $\pi^{(\mathrm{m})}$ and the one-step transition probability matrices $\left(P_{n}\right) n \geqslant m$ as the starting point. It will turn out that the structure of the tail $\sigma$-field of $\left\{x_{n}: n \geqslant m\right\}$ is crucial for the asymptotic behaviour of $\left\{\mathrm{P}_{\mathrm{m}, \mathrm{n}}\right\}$ and that $\alpha_{\mathrm{u}}^{(\mathrm{m})}(\mathrm{k})=\mathrm{P}^{(\mathrm{m})}\left(\mathrm{T}_{\mathrm{k}} \mid \mathrm{X}_{\mathrm{m}}=\mathrm{u}\right)$ where $\mathrm{T}_{\mathrm{k}}$ is an atomic set of the tail $\sigma-f i e l d$ of $\left\{x_{n}: n \geqslant m\right\}$. We first consider the countable case where a number of results are obtained under the assumption that $\lim _{\inf }{ }_{n \rightarrow \infty} P_{i, j}^{(m, n)}>0$.

A particular case is that of convergent $\left\{P_{i, j}^{(m, n)}\right\}$ where $\lim _{n \rightarrow \infty} P_{i, j}^{(m, n)}$ will be identified. Then we look at the case of finite $S$ where more powerful results are obtained without any assumption on $\left(P_{n}\right)$. Further, we specialize our 
results to some classes of finite and countable nonhomogeneous chains and explore some connections with the notion of weak ergodicity.

We do not include in this paper specific applications of products of stochastic matrices which seem to be numerous, ranging from demography as shown by Seneta [21], to recent developments in the theory of Markovian random fields assuming phase transitions (see Kemeny et a1 [12] and Wink1er [22]).

Our paper is a streamlined survey of the literature of nonhomogeneous Markov models from the viewpoint of tail $\sigma$-fields.

2. TAIL $\sigma-F I E L D S$.

Let $(\Omega, \mathcal{F}, P)$ be a probability space and $\Lambda$ a set in $\mathcal{F}$. We shall say that $\Lambda$ is a $P$-atomic set of $\mathcal{F}$ if $P(\Lambda)>0$ and $\Lambda$ does not contain any subset $\Lambda^{\prime}$ with $\Lambda^{\prime} \varepsilon \mathcal{F}$ and $0<P\left(\Lambda^{\prime}\right)<P(\Lambda)$. A nonatomic set $\Lambda$ in $\mathcal{F}$ is said to be a P-completely nonatomic set of $\mathcal{F}$ if $P(\Lambda)>0$ and $\Lambda$ does not contain any P-atomic subsets of $\mathcal{F}$. It is easy to see that, in general, $\Omega$ may be represented as $\Omega=U_{n=0}^{\infty} \Lambda_{n}$ where $\Lambda_{0}$ is P-completely nonatomic and $\Lambda_{1}, \Lambda_{2}, \ldots$ are p-atomic sets of $\mathcal{F}$. This representation is unique modulo null probability sets of $F$. Of course, some of $\left\{\Lambda_{i}\right\}$ may be absent. If $\Lambda_{0}$ is present, we shall say that $\mathcal{F}$ is nonatomic whereas if $\Lambda_{0}$ is absent $\mathcal{F}$ is called atomic. If $\Lambda_{0}$ is absent and there is only a finite number of atomic sets $\left\{\Lambda_{i}\right\}$ we shall say that $F$ is finite. Finally, $\mathcal{F}$ is said to be trivial if $\Lambda_{1}=\Omega$.

Take now $\Omega=s \times s \times \ldots$ where $s$ is finite or countable, $x_{n}(\omega)=\omega_{n}$ for $\omega=\left(\omega_{1}, \ldots, \omega_{n}, \ldots\right)$ and write $\mathcal{F}_{n}$ for the $\sigma$-field generated by $\left\{x_{k}: k \geqslant n\right\}$. A strictly positive distribution $\pi^{(\mathrm{m})}=\left(\pi_{i}^{(\mathrm{m})} ; i \varepsilon S\right)$ and a sequence of $\mathrm{S} \times \mathrm{S}$ stochastic matrices $\left(P_{n}\right)_{n} \geqslant m$ uniquely determine a probability measure $P^{(m)}$ on $\mathcal{F}_{\mathrm{m}}$ such that $\left\{\mathrm{x}_{\mathrm{n}}: \mathrm{n} \geqslant \mathrm{m}\right\}$ is a nonhomogeneous Markov chain on $\left(\Omega, \mathcal{F}_{\mathrm{m}}, \mathrm{P}^{(\mathrm{m})}\right)$ with $P^{(m)}\left(x_{m}=i\right)=\pi_{i}^{(m)}$ and $P^{(m)}\left(x_{n+1}=j \mid x_{n}=i\right)=p_{i, j}^{(n)}$ for $i, j \in S$ and $n \geqslant m$. This model will allow us to use probabilistic arguments on all $P_{i, j}^{(m, n)}$, since the equality $P_{i, j}^{(m, n)}=P^{(m)}\left(x_{n}=j \mid x_{m}=i\right)$ makes sense in view of $P^{(m)}\left(x_{m}=i\right)>0$. The usual model $\left\{x_{n}: n \geqslant 0\right\}$ may not always lead to $P^{(0)}\left(x_{m}=i\right)>0$ even if we take $\pi^{(0)}$ to be strictly positive.

We shall write $\left\{A_{n}\right.$ i.o. $\}$ for $n_{n=1}^{\infty} U_{m=n}^{\infty} A_{m}$ and $\left\{A_{n}\right.$ ult. $\}$ for $U_{n=1}^{\infty} n_{m=n}^{\infty} A_{m}$. Here 'i.o.' stands for 'infinitely often' and 'ult.' stands for 'ultimately'. Further, $\lim _{n \rightarrow \infty} A_{n}=A$ a.s. will mean that $\lim _{n \rightarrow \infty} 1_{A_{n}}=1_{A}$ a.s. where 1 stands for the indicator function of a set. We shall say that $A=B$ a.s. if $1_{A}=1_{B}$ a.s. The $\sigma$-field generated by $x_{m}, \ldots, x_{n}$ will be denoted by $F_{m}^{(n)}$.

A key tool for out study will be provided by the tail $\sigma$-field of $\left\{x_{n}: n \geqslant m\right\}$ defined as $\mathcal{J}^{(m)}=n_{n=m}^{\infty} F_{n}$.

PROPOSITION 2.1. Suppose that $\Lambda$ is a set in $\mathcal{J}^{(\mathrm{m})}$. Then there exists a sequence $\left\{L_{n}\right\}$ of subsets of $S$ such that $\lim _{n \rightarrow \infty}\left\{X_{n} \in L_{n}\right\}=\Lambda$ a.s. with respect to $\mathrm{p}^{(\mathrm{m})}$.

PROCF. Since $\Lambda$ belongs to $\mathcal{F}_{\mathrm{m}}$, the martingale convergence theorem implies that $\lim _{n \rightarrow \infty} P^{(m)}\left(\Lambda \mid \mathcal{F}_{m}^{(n)}\right)=1 \Lambda^{a} \cdot s$. with respect to $P^{(m)}$. By the Markov property of $\left\{x_{n}: n \geqslant m\right\}$ we get $P^{(m)}\left(\Lambda \mid \mathcal{F}_{m}^{(n)}\right)=P^{(m)}\left(\Lambda \mid x_{n}\right)$. Thus taking

$L_{n}=\left\{i: P^{(m)}\left(\Lambda \mid x_{n}=i\right)>\lambda\right\}$ with $0<\lambda<1$ yields $\lim _{n \rightarrow \infty}\left\{x_{n} \varepsilon L_{n}\right\}=\Lambda P^{(m)}$ a.s., completing the proof. 
The argument used above goes back, essentially, to Blackwell [1].

\section{POSITIVE STATES.}

Consider the Markov chain $\left\{x_{n}: n \geqslant m\right\}$ with a strictly positive initial probability vector $\pi^{(m)}$ and write $\pi_{j}^{(n)}=P\left(x_{n}=j\right)$ for $n \geqslant m$. We shall say that $j$ is positive if $\lim$ inf ${ }_{n \rightarrow \infty} \pi_{j}^{(n)}>0$.

PROPOSITION 3.1. A state $j$ is positive if and only if for any subsequence $\left(n_{k}\right)$ with $\lim _{n \rightarrow \infty} n_{k}=\infty$ there exists a state $i$ (possibly depending on $\left(n_{k}\right)$ ) such that $\lim \sup _{k \rightarrow \infty} P_{i, j}^{\left(m, n_{k}\right)}>0$.

PROOF. Since $\pi_{j}^{(n)}=\sum_{i \in S} \pi_{i}^{(m)} P_{i, j}^{(m, n)}$ and $\pi_{i}^{(m)}>0$ for $i \varepsilon s$, it suffices to notice that $\lim _{k \rightarrow \infty} \pi_{j}\left(n_{k}\right)=0$ if and only if $\lim _{k \rightarrow \infty} P_{i, j}\left(m_{k} n_{k}=0\right.$ for all $i \varepsilon$ s.

Proposition 3.1 shows that the definition of positivity for $j$ depends only on $\{P \underset{i, j}{(m, n)} ; n \geqslant m$, $i \varepsilon s\}$.

THEOREM 3.1. If $j$ is positive then

$$
\left\{\mathrm{X}_{\mathrm{n}}=\mathrm{j} \text { i.o. }\right\}=\mathrm{U}_{\mathrm{k}=1}^{\mathrm{d}} \mathrm{T}_{\mathrm{k}} \quad \mathrm{P}^{(\mathrm{m})} \text { a.s. }
$$

where $T_{k}, k=1, \ldots, d$ are $P^{(m)}$-atomic sets of $J^{(m)}$ with $d<\infty$.

PROOF. Notice first that $\left\{x_{n}=j i .0.\right\} \varepsilon \mathcal{J}^{(m)}$. Assume by way of contradiction that $\left\{x_{n}=j\right.$ i.o. $\}$ does not equal a finite union of atomic sets and therefore we may find some infinite sequence of disjoint sets $\mathrm{T}_{1}, \mathrm{~T}_{2}, \ldots \varepsilon \mathcal{J}^{(\mathrm{m})}$ with $\mathrm{P}^{(\mathrm{m})}\left(\mathrm{T}_{\mathrm{i}}\right)>0$ for all $\mathrm{i}$ such that

$$
\left\{x_{n}=j \text { i.o. }\right\}=\bigcup_{k=1}^{\infty} T_{k} \quad P^{(m)} \text { a.s. }
$$

Take $\delta=1 \mathrm{im} \inf _{n \rightarrow \infty} \pi_{j}^{(n)}$. If (3.1) were true, there would exist a set $T_{k}$ with $\mathrm{P}^{(\mathrm{m})}\left(\mathrm{T}_{\mathrm{k}}\right)<\delta$. Let $\left\{\mathrm{E}_{\mathrm{n}}^{(\mathrm{k})}: \mathrm{n} \geqslant \mathrm{m}\right\}$ be some subsets of $\mathrm{S}$ such that $\lim _{\mathrm{n} \rightarrow \infty}\left\{\mathrm{X}_{\mathrm{n}} \varepsilon \mathrm{E}_{\mathrm{n}}^{(\mathrm{k})}\right\}=\mathrm{T}_{\mathrm{k}} \quad \mathrm{P}^{(\mathrm{m})}$ a.s. as ensured by Proposition 3.1. In view of (3.1) $j$ must belong to an infinity of sets $\left\{E_{n}^{(k)}\right\}$. This entails

$$
\underset{n \rightarrow \infty}{\lim \inf } \pi_{j}^{(n)} \geqslant \lim _{n \rightarrow \infty} P^{(m)}\left(E_{n}^{(k)}\right)=P^{(m)}\left(T_{k}\right)<\delta
$$

which is absurd. Thus $\left\{x_{n}=j i . o_{0}\right\}$ consists of a finite number of $P^{(m)}$-atomic sets of $\mathcal{J}^{(\mathrm{m})}$.

THEOREM 3.2. Suppose that $j$ is positive. Then there exist $d$ disjoint sequences of integers $\left\{\Gamma_{k}\right\}$ where $\Gamma_{k}=\left\{n_{t}^{(k)}: t=1,2, \ldots\right\}$ and $d$ sequences of sets $\left\{E_{n}^{(k)}\right\}$ such that $U_{k=1}^{d} \Gamma_{k}=\{m+1, \ldots\}$, and for $i, l \in S$

$$
\lim _{\substack{n^{\prime} \rightarrow \infty \\ n^{\prime} \varepsilon \Gamma_{k}}} P_{i, j}^{\left(m, n^{\prime}\right)} / P_{\ell, j}^{\left(m, n^{\prime}\right)}=\alpha_{i}^{(m)}(k) / \alpha_{\ell}^{(m)}(k)
$$

provided that $P_{l, j}^{\left(m, n^{\prime}\right)}>0$ where $\alpha_{u}^{(m)}(k)=1 i m_{n \rightarrow \infty} \sum_{j \in E_{n}}(k) P_{u, j}^{(m, n)}$ for $u \varepsilon s$. 
PROOF. Let $\left\{\mathrm{E}_{\mathrm{n}}^{(\mathrm{k})}\right\}$ be some sequences of sets with $\lim _{\mathrm{n} \rightarrow \infty}\left\{\mathrm{X}_{\mathrm{n}} \in \mathrm{E}_{\mathrm{n}}^{(\mathrm{k})}\right\}=\mathrm{T}_{\mathrm{k}}$ $\mathrm{P}^{(\mathrm{m})}$ a.s. for $k=1,2, \ldots, \mathrm{d}$ whose existence is ensured by Proposition 2.1. We first show that $j \in U_{k=1}^{d} E_{n}^{(k)}$ for $n$ sufficiently large.

Indeed, if we assume the contrary, i.e. that $j \notin U_{k=1}^{d} E_{n}^{(k)}$ for a sequence $\left\{n_{k}\right\}$ with $\lim _{t \rightarrow \infty} n_{t}=\infty$, the positivity of $j$ leads to $P^{(m)}\left(x_{n_{t}}=j i .0_{.}\right)>0$ and contradicts $\left\{x_{n} \in U_{k=1}^{d} E_{n}^{(k)} i . o_{0}\right\}=\left\{x_{n} \varepsilon U_{k=1}^{d} E_{n}^{(k)} u 1 t.\right\}=U_{k=1}^{d} T_{k} P^{(m)}$ a.s. Thus, we may assume, if necessary by modifying a finite number of sets $\left\{\mathrm{E}_{\mathrm{n}}^{(\mathrm{k})}\right\}$, that $j \in U_{k=1}^{d} E_{n}^{(k)}$ for $n=m+1, \ldots$

Define now the random variable $P_{i, X_{n}}^{(m, n)} / P_{\ell, X_{n}}^{(m, n)}$ to equal $P_{i, u}^{(m, n)} / P_{\ell, u}^{(m, n)}$ for $\omega \varepsilon\left\{\mathrm{X}_{\mathrm{n}}=\mathrm{u}\right\}$ if $\mathrm{P}_{\ell, \mathrm{u}}^{(\mathrm{m}, \mathrm{n})}>0$ and 0 otherwise. Since

$$
P_{i, u}^{(m, n)} / P_{\ell, u}^{(m, n)}=\frac{P^{(m)}\left(x_{m}=i \mid x_{n}=u\right) \pi_{l}^{(m)}}{P^{(n)}\left(x_{m}=\ell \mid x_{n}=u\right) \pi_{i}^{(m)}}
$$

the Markov property of the reversed chain yields

$$
\lim _{n \rightarrow \infty} P_{i, x_{n}}^{(m, n)} / P_{\ell, x_{n}}^{(m, n)}=\frac{P^{(m)}\left(x_{m}=i F_{m}^{(n)}\right)}{P^{(m)}\left(x_{m}=\ell F_{m}^{(n)}\right)} \frac{\pi_{l}^{(m)}}{\pi_{i}^{(m)}}
$$

Using the martingale convergence theorem and Theorem 2.1 in (3.4) leads to

$$
\lim _{n \rightarrow \infty} P_{i, X_{n}}^{(m, n)} / P_{l, X_{n}}^{(m, n)}=\frac{P^{(m)}\left(X_{m}=i \mid T_{k}\right)}{P^{(m)}\left(X_{m}=\ell \mid T_{k}\right)} \frac{\pi_{l}^{(m)}}{\pi_{i}^{(m)}}=\frac{P^{(m)}\left(T_{k} \mid x_{m}=i\right)}{P^{(m)}\left(T_{k} \mid X_{m}=l\right)}
$$

for almost all $\omega \in T_{k}, k=1, \ldots, 2$, provided that $P^{(m)}\left(x_{m}=\ell \mid T_{k}\right)>0$. Notice that $P^{(m)}\left(T_{k} \mid x_{m}=u\right)=1 \lim _{n \rightarrow \infty} P^{(m)}\left(X_{n} \in E_{n}^{(k)} \mid x_{m}=u\right)=1 \lim _{n \rightarrow \infty} \sum_{j \in E_{n}}(k) P_{u, j}^{(m, n)}=\alpha_{u}^{(m)}(k)$. Let $\left\{n_{t}^{(k)}: t=1,2, \ldots\right\}$ be the set of values of $n$ with $j \varepsilon E_{n}^{(k)}$. We show now that (3.5) holds if $n$ runs through $\left\{n_{t}^{(k)}: t=1, \ldots\right\}$ and $x_{n}$ is replaced by $j$ - Indeed, suppose the contrary. Then there exists $\left\{n_{t}\right\} \subseteq\left\{n_{t}^{(k)}\right\}$ with $\lim _{t \rightarrow \infty} n_{t}=\infty$ such that

$$
\lim _{t \rightarrow \infty} P_{i, j}^{(m, n)} / P_{l, j}^{\left(m, n_{t}\right)} \neq \frac{P^{(m)}\left(T_{k} \mid x_{m}=i\right)}{P(m)\left(T_{k} \mid x_{m}=l\right)}
$$

for some $k \varepsilon\{1, \ldots, d\}$. But $\left\{x_{n_{t}}=j i .0.\right\} \subseteq T_{k}$ a.s.

$P^{(m)}\left(\left\{x_{n_{t}}=j i .0.\right\}\right) \geqslant \lim \inf _{n \rightarrow \infty} \pi_{j}^{(n)}>0$ makes (3.6) contradict (3.5) and completes the proof.

REMARK 3.1. Theorem 3.2 was stated as a result about products of stochastic matrices without reference to Markov chains theory. We have seen in the proof that $\alpha_{\mathrm{u}}^{(\mathrm{m})}(\mathrm{k})$ may be expressed in terms of the tail $\sigma-f$ ield 
$\mathcal{J}^{(\mathrm{m})}$ as $\mathrm{P}^{(\mathrm{m})}\left(\mathrm{T}_{\mathrm{k}} \mid \mathrm{x}_{\mathrm{m}}=\mathrm{u}\right)$. We shall further consider some characteristic properties of the sets $\left\{E_{n}^{(k)}\right\}$ which, for some special cases, may lead to the actual identification of $\left\{\alpha_{\mathrm{u}}^{(\mathrm{m})}(\mathrm{k})\right\}$.

THEOREM 3.3. Suppose that $j$ is positive and $\left\{x_{n}=j\right.$ i.o. $\}=U_{k=1}^{d} T_{k} P^{(m)}$ a.s. where $\left\{T_{k}\right\}$ are $P^{(m)}$-atomic sets of $\mathcal{J}^{(m)}$, and let $\left\{E_{n}^{(k)}\right\}$ be some sets such that $\lim _{n \rightarrow \infty}\left\{x_{n} \varepsilon E_{n}^{(k)}\right\}=T_{k} P(m)$ a.s. Let $\Gamma_{k}=\left\{n_{k}^{(t)}\right\}$ be the set of values $n$ such that $j \in E_{n}^{(k)}, k=1, \ldots, d$. Then

$$
\sum_{n \in \Gamma_{k}} \sum_{i \notin E}(k) p_{i, j}^{(n)}<\infty
$$

PROOF。 Define $A_{n}=\left\{x_{n}=j, X_{n+1} \notin E_{n+1}^{(k)}\right\}$ for $n \geqslant m$. It is clear that

$$
{ }^{(m)}\left(A_{n}, n \in \Gamma_{k} \text { i.o. }\right)=0 \text {. }
$$

Recall $\mathcal{F}_{m}^{(n)}$ is the $\sigma$-field generated by $x_{m}, \ldots, x_{n}$ with $m<n$. According to the Bore1-Cantelli-Levy lemma

$$
\begin{aligned}
& P^{(m)}\left(A_{n}, n \in \Gamma_{k} \text { i.o. }\right)=0 \text { if and only if } \\
& \qquad P^{(m)}\left(\sum_{n \in \Gamma_{k}}{ }^{(m)}\left(A_{n} \mid F_{m}^{(n)}\right)=\infty\right)=0 .
\end{aligned}
$$

Notice now that the Markov property of $\left\{x_{n}: n \geqslant m\right\}$ yields

$$
P^{(m)}\left(A_{n} \mid \mathcal{F}_{m}^{(n)}\right)=P^{(m)}\left(x_{n+1} \notin E_{n+1}^{(k)} \mid x_{n}=j\right) 1_{\left\{x_{n}=j\right\}} \text {. }
$$

Thus, it will suffice to show that $P^{(m)}\left(\sum_{n \in \Gamma_{k}}{ }^{(m)}\left(A_{n} \mid F_{m}^{(n)}\right)=\infty\right)=0$ implies (3.7). Assume, by way of contradiction, that

$$
\sum_{n \in \Gamma_{k} i \notin E_{n+1}^{(k)}} P_{j, i}^{(n)}=\sum_{n \in \Gamma_{k}} P^{(m)}\left(x_{n+1} \notin E_{n+1}^{(k)} \mid x_{n}=j\right)=\infty
$$

and write

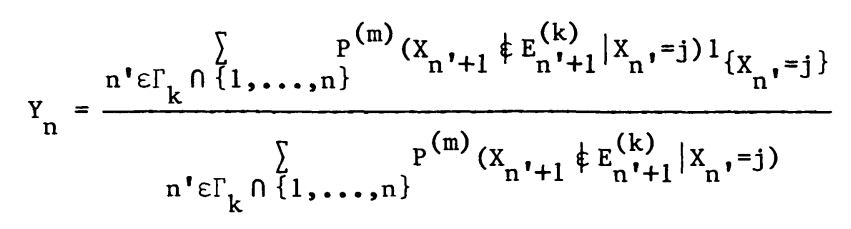

Since $0 \leqslant Y_{n} \leqslant 1$, for $\left\{Y_{n}\right\}$ to converge in probability to 0 as $n \rightarrow \infty$, it is necessary that $E^{(m)}\left(Y_{n}\right) \rightarrow 0$ as $n \rightarrow \infty$, where $E^{(m)}\left(Y_{n}\right)$ denotes the expectation of $Y_{n}$ under $P^{(m)}$. However, $1 \mathrm{im} \inf _{n \rightarrow \infty} E^{(m)}\left(Y_{n}\right) \geqslant 1 \lim \inf _{n \rightarrow \infty} \pi_{j}^{(n)}>0$ and therefore $P^{(m)}\left(\lim \sup _{n \rightarrow \infty} Y_{n}>0\right)>0$. Since the denominator of $Y_{n}$ in $(3.12)$ tends to $\infty$ as $\mathrm{n} \rightarrow \infty$, it follows that the numerator of $\mathrm{Y}_{\mathrm{n}}$ will tend to $\infty$ as $\mathrm{n} \rightarrow \infty$ on a set of positive $\mathrm{P}^{(\mathrm{m})}$ probability. Taking account of $(3.9)$ and $(3.10)$ yields $\mathrm{P}^{(\mathrm{m})}\left(\sum_{\mathrm{n} \in \Gamma_{\mathrm{k}}}{ }^{(\mathrm{m})}\left(\mathrm{A}_{\mathrm{n}} \mid \mathcal{F}_{\mathrm{m}}^{(\mathrm{n})}\right)=\infty\right)>0$, a contradiction that completes the proof. 
REMARK 3.2. For homogeneous Markov chains with period $d$, the sets $\left\{\mathrm{E}_{\mathrm{n}}^{(\mathrm{k})}\right\}$ become $\left\{\mathrm{C}_{\mathrm{u}+n(\bmod d)}\right\}$, i.e. the cyclically moving subclasses of the recurrent class to which $j$ belongs. In this case

$\left.\mathrm{P}^{(\mathrm{m})}\left(\left\{\mathrm{X}_{\mathrm{n}} \in \mathrm{C}_{\mathrm{u}+\mathrm{n}(\bmod \mathrm{d})}\right\} \mid \mathrm{x}_{\mathrm{n}-1} \in \mathrm{C}_{\mathrm{u}+\mathrm{n}-1(\bmod \mathrm{d})}\right\}\right)=1$,

$\lim _{n \rightarrow \infty}\left\{x_{n} \varepsilon C_{u+n(\bmod d)}\right\}=T_{u}, u=1, \ldots, d$, and (3.7) holds trivially in view of $p_{j, i}^{(n)}=0$ for $i \notin C_{u+n+1(\bmod d)}$.

The results and proofs of this Section rely on Cohn [5], [6] and [8].

4. A Class OF COUNTABLE CHAINS.

We shall next consider a class of stochastic matrices $\left(P_{n}\right)$ satisfying the following condition

(A) $S$ admits the decomposition $S=T \cup C$ where $j \varepsilon T$ if $\lim _{n \rightarrow \infty} P_{i, j}^{(m, n)}=0$ for all $m$ and $i$, and $j \in C$ if there exists $m$ such that $j$ is positive for $\left\{x_{n}: n \geqslant m\right\}$.

A partition $T, C_{1}, C_{2}, \ldots$ of $S$ will be said to be a basis for $\left(P_{n}\right)$ if for any $j \in C_{k}$ and $m$ large enough, $\left\{x_{n}=j\right.$ i.o. $\}=T_{k}$ a.s. is a $P^{(m)}$-atomic set of $J^{(m)}$

THEOREM 4.1. Suppose that $\left(P_{n}\right)$ satisfies condition $(A)$. Then the existence of $\lim _{n \rightarrow \infty} P_{i, j}^{(m, n)} / P_{\ell, j}^{(m, n)}$ for $i, l \varepsilon S, m \geqslant 0$ and $j \varepsilon C$ with $P_{\ell, j}^{(m, n)}>0$ is a necessary and sufficient condition for the existence of a basis $T$, $\mathrm{c}_{1}, \mathrm{C}_{2}, \ldots$.

PROOF. We know from Proposition 3.1 that $\Lambda=\left\{x_{n}=j\right.$ i.o. $\}$ is a finite union of $P^{(m)}$-atomic sets of $J^{(m)}$, i.e. $\Lambda=U_{k=1}^{d} T_{k}$. We shall first prove that $d=1$. Suppose otherwise, i.e. $d \geqslant 2$. Since $\left\{x_{n}=j\right.$ i.o. $\} \supseteq T_{1} \cup T_{2}$, Theorem 3.1 implies that there must exist two sequences $\left\{n_{k}\right\}$ and $\left\{n_{k}^{\prime}\right\}$ such that

$$
\begin{aligned}
& \lim _{k \rightarrow \infty} P_{i, j}^{\left(m, n_{k}\right)} / P_{\ell, j}^{\left(m, n_{k}\right)}=P\left(T_{1} \mid x_{m}=i\right) / P\left(T_{1} \mid x_{m}=\ell\right) \\
& \lim _{k \rightarrow \infty} P_{i, j}\left(m, n_{k}\right) / P_{\ell, j}^{\left(m, n_{k}\right)}=P\left(T_{2} \mid x_{m}=i\right) / P\left(T_{2} \mid x_{m}=\ell\right)
\end{aligned}
$$

However, $\lim _{n \rightarrow \infty} P_{i, j}^{(m, n)} / P_{\ell, j}^{(m, n)}$ was supposed to exist. Thus (4.1) entails

$$
\mathrm{P}^{(\mathrm{m})}\left(\mathrm{T}_{1} \mid \mathrm{x}_{\mathrm{m}}=\mathrm{i}\right) / \mathrm{P}^{(\mathrm{m})}\left(\mathrm{T}_{1} \mid \mathrm{x}_{\mathrm{m}}=l\right)=\mathrm{P}^{(\mathrm{m})}\left(\mathrm{T}_{2} \mid \mathrm{x}_{\mathrm{m}}=\mathrm{i}\right) / \mathrm{P}^{(\mathrm{m})}\left(\mathrm{T}_{2} \mid \mathrm{x}_{\mathrm{m}}=\ell\right)
$$

Further, for any set $A$ in $\mathcal{T}^{(m)}$ with $P^{(m)}(A)>0$, the martingale convergence theorem in conjunction with the time reversibility of the Markov property

$$
\mathrm{P}^{(\mathrm{m})}\left(\mathrm{A} \mid \mathrm{X}_{\mathrm{n}}\right) \text { may be made as close as desired to } 1_{A} \text { for } n
$$

large enough. It is easy to see that if $j$ is positive for $\left\{x_{n}: n \geqslant m\right\}$ it will stay positive for any $\left\{x_{n}: n \geqslant m^{\prime}\right\}$ with $m^{\prime}>m$, and $P^{(m)}\left(A \mid x_{n}=j\right)=P^{\left(m^{\prime}\right)}\left(A \mid x_{n}=j\right)$ if $P^{(m)}\left(X_{n}=j\right) P^{\left(m^{\prime}\right)}\left(x_{n}=j\right)>0$. Thus, one may choose $i$, $\ell$ and $m$ such that for $\varepsilon<\frac{1}{2} P^{(m)}\left(T_{1} \mid x_{m}=i\right)>1-\varepsilon$ and 
$\mathrm{P}^{(\mathrm{m})}\left(\mathrm{T}_{2} \mid \mathrm{x}_{\mathrm{m}}=\ell\right)>1-\varepsilon$; since $\mathrm{T}_{1}$ and $\mathrm{T}_{2}$ are disjoint we also get $\mathrm{P}^{(\mathrm{m})}\left(\mathrm{T}_{2} \mid \mathrm{X}_{\mathrm{m}}=\mathrm{i}\right)<\varepsilon$ and $\mathrm{P}^{(\mathrm{m})}\left(\mathrm{T}_{1} \mid \mathrm{X}_{\mathrm{m}}=\ell\right)<\varepsilon$. It is easy to see that these four inequalities are in contradiction with (4.2). We have reached a contradiction that proves that $\Lambda=\left\{x_{n}=j i . o.\right\}$ is a $P^{(m)}$-atomic set of $J^{(m)}$.

Conversely, if we assume that $\Lambda=\left\{x_{n}=j i .0.\right\}$ is a $P^{(m)}$-atomic set of $\mathcal{J}^{(m)}$, then Theorem 3.2 yields that $1 \lim _{n \rightarrow \infty} P_{i, j}^{(m, n)} / P_{\ell, j}^{(m, n)}=P^{(m)}\left(\Lambda \mid x_{m}=i\right) / P^{(m)}\left(\Lambda \mid x_{m}=l\right)$, completing the proof.

THEOREM 4.2. Suppose that $\left(\mathrm{P}_{\mathrm{n}}\right)$ assumes a basis $\mathrm{T}, \mathrm{C}_{1}, \mathrm{C}_{2}, \ldots$. Then (i) for any $j \in C_{k}$, i, $\ell \in S$ with $P_{\ell, j}^{(m, n)}>0$ and $n$ large enough

$$
\lim _{n \rightarrow \infty} P_{i, j}^{(m, n)} / P_{\ell, j}^{(m, n)}=\alpha_{i}^{(m)}(k) / \alpha_{\ell}^{(m)}(k)
$$

where $\alpha_{u}^{(m)}(k)=1 i m_{n \rightarrow \infty} L_{j \in E_{n}}^{(k)} P_{u, j}^{(m, n)}$ with 1 im $\sup _{n \rightarrow \infty} \sum_{j \in C_{k}} P_{u, j}^{(m, n)} \leqslant \alpha_{u}^{(m)}(k)$.

(ii) if for any $C_{k}$ there exists $\lambda$ with $0<\lambda<1$ such that $\alpha_{i}^{(m)}(k)<1-\lambda$ for $j \varepsilon C_{k}$, with $k^{\prime} \neq k$ and $m$ large enough, then $i \varepsilon C_{k}$ implies

$$
\sum_{n=1}^{\infty} \sum_{i \notin C_{k}} \mathrm{p}_{\mathrm{T}}^{(\mathrm{n})}{ }_{j, i}<\infty
$$

PROOF. Part (i) follows from Theorem 4.1 and Theorem 3.2 if we notice that $\lim \sup _{n \rightarrow \infty} \sum_{j \in C_{k}} P_{u, j}^{(m, n)} \leqslant \lim _{n \rightarrow \infty} \sum_{j \in E}(k) P_{u, j}^{(m, n)}$. Indeed, although it is not true in general, that $\mathrm{P}^{(\mathrm{m})}\left(\mathrm{X}_{\mathrm{n}} \varepsilon \mathrm{C}_{\mathrm{k}}\right.$ i.o. $)=\mathrm{P}^{(\mathrm{m})}\left(\mathrm{T}_{\mathrm{k}}\right),\left\{\mathrm{X}_{\mathrm{n}} \in \mathrm{F}\right.$ i.o. $\}=\mathrm{T}_{\mathrm{k}}$ $\mathrm{P}^{\text {(m) }}$ a.s. obtains for any finite set $\mathrm{F} \subset \mathrm{C}_{\mathrm{k}}$. This being true for any $\mathrm{k}$ and the states of $\mathrm{C}_{\mathrm{k}}$ being positive necessarily imply that $\mathrm{F} \subset \mathrm{E}_{\mathrm{n}}^{(\mathrm{k})}$ for $\mathrm{n}$ large enough and therefore $\lim _{n \rightarrow \infty} j \in C_{k}{ }_{u, j}^{(m, n)} \leqslant \alpha_{u}^{(m)}(k)$, which proves (i).

For part (ii) we may invoke Theorem 3.3 provided that we show that $C_{k} \cup T \supseteq E_{n}^{(k)}$. The latter follows from taking $E_{n}^{(k)}=\left\{j: P^{(m)}\left(T_{k} \mid x_{n}=j\right)<\lambda\right\}$, noticing that, as shown before, $\left.P^{(m)}\left(T_{k} \mid x_{n}=i\right)=1 \operatorname{im}_{n \rightarrow \infty} P^{(m)}\left(x_{n} \in E_{n}^{(k)}\right\} \mid x_{n}=i\right)$ and arguing as in the proof of Proposition 1.1 .

REMARK 4.1. It is easy to see that in case that $\lim _{n \rightarrow \infty} \sum_{j \in T} P_{u, j}^{(m, n)}=0$ for a11 $u \in S$ we get that $\alpha_{u}^{(m)}(k)=\lim _{n \rightarrow \infty} \sum_{j \varepsilon C_{k}}{ }_{u, j}^{(m, n)}$. This happens when $\lim _{n \rightarrow \infty} P^{(m)}\left(X_{n} \varepsilon T\right)=0$. In particular, the finite chains always satisfy this condition.

The results in this Section were derived in Cohn [8].

5. CONVERGENT CHAINS.

A chain $\left\{\mathrm{x}_{n}\right\}$ will be said to be convergent if $\lim _{n \rightarrow \infty} \mathrm{P}_{i, j}^{(m, n)}$ exists for all $m$, $i$ and $j$ (see Maksimov [15], Iosifescu [11], and Mukherjea [17]). Of course, this definition depends only on $\left(P_{n}\right)$ and does not need to involve a 
chain $\left\{x_{n}\right\}$. As before our results may be read off without reference to a Markov chain structure. The class of matrices $\left(P_{n}\right)$ for which $\left\{P_{i, j}^{(m, n)}\right\}$ converge is a subclass of that considered in Section 4 and, naturally, will lead to stronger properties. For convergence chains we shall identify the limits of $\{\mathrm{P} \underset{i, j}{(\mathrm{~m}, \mathrm{n})}\}$ rather than those of their ratios as was done in the previous sections. THEOREM 5.1. Suppose that $\left\{\mathrm{x}_{\mathrm{n}}\right\}$ is a convergent chain. Then

(i) there exists a basis $\left\{\mathrm{T}, \mathrm{C}_{1}, \mathrm{C}_{2}, \ldots\right\}$

(ii) for any $m, i \varepsilon S$ and $j \varepsilon C_{k}$

$$
\lim _{n \rightarrow \infty} P_{i, j}^{(m, n)}=\pi_{j} \alpha_{i}^{(m)}(k) / \alpha_{k}
$$

where $\alpha_{i}^{(m)}(k)=\lim _{n \rightarrow \infty} \sum_{j \in E_{n}^{(k)}} P_{i, j}^{(m, n)}, \alpha_{k}=\lim _{n \rightarrow \infty} \sum_{j \in E_{n}^{(k)}} \pi_{j}^{(n)}$ and $\pi_{j}=1 \lim _{n \rightarrow \infty} \pi_{j}^{(n)}$ with $\pi_{j}^{(n)}=\sum_{i \varepsilon S} \pi_{i}^{(m)} P_{i, j}^{(m, n)}, n \geqslant m$.

REMARK 5.1. Since $\left\{P_{i, j}^{(m, n)}\right\}$ do not depend on the choice of $\pi^{(m)}$ provided that $\pi_{i}^{(m)}>0$, we notice that $\pi_{j} / \alpha_{k}$ is also indepedent of $\pi^{(m)}$.

PROOF. Theorem 4.1 ensures the existence of a basis $\left\{T_{,} C_{1}, C_{2}, \ldots\right\}$. Further, the martingale convergence theorem yields

$$
P^{(m)}\left(X_{m}=i \mid X_{n}\right)=P^{(m)}\left(X_{m}=i \mid \mathcal{F}_{m}^{(n)}\right) \rightarrow P^{(m)}\left(X_{m}=i \mid \mathcal{J}^{(m)}\right) \text { as } n \rightarrow \infty \text {. }
$$

But

$$
P^{(m)}\left(x_{m}=i \mid x_{n}=j\right)=P_{i, j}^{(m, n)} \pi_{i}^{(m)} / \pi_{j}^{(n)}
$$

and taking into account that $\left\{x_{n}=j i .0.\right\}=T_{k}$ a.s. is a $P^{(m)}$-atomic set of $\mathcal{J}^{(\mathrm{m})}$ we get

$$
\lim _{n \rightarrow \infty} \pi_{i}^{(m)} P_{i, x_{n}}^{(m, n)} / \pi_{X_{n}}^{(n)}=P^{(m)}\left(x_{m}=i \mid T_{k}\right)
$$

for almost all $\omega \in \mathrm{T}_{\mathrm{k}}$. Further we can argue as in the proof of Theorem 4.2(i) to conclude (ii) on using (5.1).

COROLLARY 5.1. Suppose that $\left\{\mathrm{X}_{\mathrm{n}}\right\}$ is a countable convergent chain assuming a basis $\left\{T, C_{1}, C_{2}, \ldots\right\}$ and denote $q_{i, j}^{(m)}=\lim _{n \rightarrow \infty} P_{i, j}^{(m, n)}$ and $q_{i, j}=1 i m_{m \rightarrow \infty} q_{i, j}^{(m)}$. Then

$$
q_{i, j}=\left\{\begin{array}{l}
\pi_{j} / \alpha_{k} \text { for } i \varepsilon C_{k}, j \varepsilon C_{k} \\
0
\end{array}\right.
$$

PROOF. According to Theorem 5.1, $q_{i, j}^{(m)}=\pi_{j} \alpha_{i}^{(m)}(k) / \alpha_{k}$ and since $i \varepsilon C_{k}$ $\operatorname{im}_{m \rightarrow \infty} \pi_{i}^{(m)}>0$ and $\left\{X_{n}=i\right.$ i.o. $\}=T_{k} P^{(m)}$ a.s. we easily conclude that $\mathrm{P}^{(\mathrm{m})}\left(\mathrm{T}_{\mathrm{k}} \mid \mathrm{X}_{\mathrm{m}}=\mathrm{i}\right) \rightarrow 1$ as $\mathrm{m} \rightarrow \infty$, and the case $i \varepsilon \mathrm{C}_{k}$ and $j \varepsilon \mathrm{C}_{k}$ follows. Assume now that $j \notin C_{k}$. Then either $j \varepsilon T$ which yields $q_{i j}=0$ for $i \varepsilon C_{k}$, or $j \varepsilon C_{k^{\prime}}$, with $k^{\prime} \neq k$, in which case $P^{(m)}\left(T_{k}, \mid x_{n}=i\right) \rightarrow 0$ as $n \rightarrow \infty$ is a consequence of 
$\mathrm{P}^{(\mathrm{m})}\left(\mathrm{T}_{\mathrm{k}} \mid \mathrm{X}_{\mathrm{m}}=\mathrm{i}\right) \rightarrow 1$ as $\mathrm{m} \rightarrow \infty$ and $\mathrm{T}_{\mathrm{k}}$ and $\mathrm{T}_{\mathrm{k}}$, being disjoint. Using now Theorem 5.1 (ii) yields $q_{i j}=0$ and completes the proof.

REMARK 5.2. Theorem 4.2(ii) holds, of course, for convergent chains as well. The additional condition imposed on convergent chains does not seem to make this result any stronger.

The convergent chain concept goes back to Maksimov [15], who considered the case of bistochastic matrices $\left(P_{n}\right)$. Extensions to finite and countable convergent chains were given by Mukherjea [16-18], etc. The methods used in these papers are matricial and the sets of the basis are characterized by means of some limit points of matrices $\left\{Q_{k}\right\}$ with $Q_{k}=1 i m_{n \rightarrow \infty} P_{k, n}$. However, such matricial methods yielded much weaker results and do not seem to permit the identification of $\left\{\mathrm{Q}_{\mathrm{k}}\right\}$.

The results of this section were derived in Cohn [8].

6. FINITE MARKOV CHAINS: THE GENERAL CASE.

We shall now consider the case when $s$, the number of elements of $S$, is finite. For such chains we shall derive stronger results under less restrictive or no assumptions on $\left\{\mathrm{P}_{n}\right\}$. As before we need start off by considering the structure of $J(m)$.

THEOREM 6.1. The tail $\sigma$-field $J^{(m)}$ of $\left\{x_{n}: n \geqslant m\right\}$ is finite and the number of $\mathrm{P}^{(\mathrm{m})}$-atomic sets of $\mathcal{J}^{(\mathrm{m})}$ does not exceed $\mathrm{s}$.

PROOF. Let $T_{1}, \ldots, T_{d}$ be some disjoint sets in $\mathcal{J}^{(m)}$ with $P^{(m)}\left(T_{k}\right)>0$ for $k=1, \ldots, d$. As shown in the proof of Proposition 1.1 , letting $E_{n}^{(k)}=\left\{j: P^{(m)}\left(T_{k} \mid x_{n}=j\right)>0.5\right\}$ yields $1 i_{n \rightarrow \infty}\left\{x_{n} \varepsilon E_{n}^{(k)}\right\}=T_{k} P^{(m)}$ a.s. for $k=1, \ldots, d$. It is easy to check that $\left\{E_{n}^{(k)}, k=1, \ldots, d\right\}$ are disjoint for any $\mathrm{n}$. Since $\mathrm{P}^{(\mathrm{m})}\left(\mathrm{T}_{\mathrm{k}}\right)>0,\left\{\mathrm{E}_{\mathrm{n}}^{(\mathrm{k})}\right\}$ must be non-empty for $\mathrm{n}$ large. However, there are $s$ states in $S$ which requires $d \leqslant s$ and completes the proof.

LEMMA 6.1. Let $\left\{X_{n}: n \geqslant m\right\}$ and $\left\{X_{n}^{\prime}: n \geqslant m^{\prime}\right\}$ be two finite Markov chains with strictly positive initial probability vectors $\pi^{(m)}$ and $\pi^{\prime(m)}$ and sequences of transition probability matrices $\left(P_{n}\right)_{n} \geqslant m$ and $\left(P_{n}\right)_{n} \geqslant m^{\prime}$ respectively. Write $\pi_{i}^{(n)}=P^{(m)}\left(X_{n}=i\right), \pi_{i}^{(n)}=P^{\left(m^{\prime}\right)}\left(X_{n}^{\prime}=i\right), E_{n}^{+}=\left\{i: \pi_{i}^{(n)}>0\right\}$ and $E_{n}^{\prime+}=\left\{i: \pi_{i}^{\prime(n)}>0\right\}$. Then there exists a number $N>0$ such that $E_{n}^{+}=E_{n}^{\prime+}$ for $m, m^{\prime} \geqslant N$ and $n \geqslant \max \left(m, m^{\prime}\right)$.

PROOF. Suppose that $\mathrm{m}^{\prime}>\mathrm{m}$ and let $j \in \mathrm{E}_{\mathrm{n}}^{+}$. Then $\pi_{j}^{(n)} \geqslant \pi_{i}^{(m)} P_{i, j}^{(m, n)}>0$ for some $i \varepsilon S$ and since $\pi_{i}^{(m)}>0$, it follows that $P_{i, j}^{(m, n)}>0$. By the Chapman-Kolmogorov formula there must exist $l \in \mathrm{S}$ such that $\mathrm{P}_{i, l}^{\left(m, m^{\prime}\right)}{ }_{P}{ }_{l, j}^{\left(m^{\prime} \cdot n\right)}>0$. Thus. $\pi_{j}^{\prime(n)} \geqslant \pi^{\prime\left(m^{\prime}\right)}{ }_{P}^{\left(m^{\prime}, n\right)}>0$. We have shown that $E_{n}^{+} \subseteq E_{n}^{{ }^{+}}$. Notice that the finiteness of $S$ makes it impossible that $E_{n}^{+} \subset E_{n}^{{ }^{+}}$with strict inclusion for all $\mathrm{m}, \mathrm{n}$ and $\mathrm{m}^{\prime}>\mathrm{m}$. Thus there must exist $\mathrm{m}$ and $\mathrm{N}$ such that for $\mathrm{m}^{\prime}>\mathrm{m}$ $\mathrm{E}_{\mathrm{N}}^{+}=\mathrm{E}_{\mathrm{N}}^{{ }^{+}}$. Choose now $\mathrm{n}>\mathrm{N}$ and $\mathrm{j} \varepsilon \mathrm{E}_{\mathrm{n}}^{\mathbf{O}^{+}}$. Then there is a state $\mathrm{i} \varepsilon \mathrm{E}_{\mathrm{N}}^{{ }^{+}}$such 
that $\pi_{j}^{\prime(n)} \geqslant \pi_{i}^{\prime(N)} P_{i, j}^{(N, n)}>0$. Thus $P_{i, j}^{(N, n)}>0$ for $i \varepsilon E_{N}^{\prime^{+}}$. But $E_{N}^{\prime^{+}}=E_{N}^{+}$leads to $\pi_{j}^{(n)} \geqslant \pi_{i}^{(N, n)}>0$ and $E_{n}^{+}=E_{n}^{\prime+}$ obtains for $n>N$, completing the proof.

THEOREM 6.2. Suppose that $\mathrm{S}$ is finite. Then there exist some sequences of subsets of $s,\left\{E_{n}^{(k)}\right\}, k=1, \ldots, s$, such that for $m=0,1, \ldots$

$$
\lim _{n \rightarrow \infty} P_{i, j}^{(m, n)} / P_{\ell, j}^{(m, n)}=\alpha_{i}^{(k)}(m) / \alpha_{\ell}^{(k)}(m)
$$

for $j \in E_{n}^{(k)}, k=1, \ldots, d$, where $\alpha_{u}^{(k)}=\lim _{n \rightarrow \infty} \sum_{j \in E_{n}^{(k)}} P_{u, j}^{(m, n)}$ provided that $\alpha_{\ell}^{(k)}>0$, and

$$
\lim _{n \rightarrow \infty} P_{i, j}^{(m, n)}=0
$$

for $j \varepsilon E_{n}=s-U_{k=1}^{d} E_{n}^{(k)}$.

REMARK. Throughout the paper we will drop the explicit dependence of $j$ upon $\mathrm{n}$; and so $\mathrm{j}$ is not kept fixed, but in general varying with $E_{n}^{(k)}$. The same convention will be valid for all the sets dependent on $n$ to be further considered.

PROOF. Choose $m$ such that the sets $\left\{E_{n}^{+}\right\}$attached to $\left\{X_{n}: n \geqslant m\right\}$ are maximal. Such a choice is always possible in view of Lemma 6.1 .

According to (3.5)

$$
\lim _{n \rightarrow \infty} P_{i, X_{n}}^{(m, n)} / P_{\ell, X_{n}}^{(m, n)}=P^{(m)}\left(T_{k} \mid x_{m}=i\right) / P^{(m)}\left(T_{k} \mid x_{m}=\ell\right)
$$

for almost all $\omega \varepsilon \mathrm{T}_{k}, k=1, \ldots$,

Write now

$$
\begin{aligned}
E_{n, k}^{(r)}=\left\{j: P\left(T_{k} \mid X_{n}=j\right)>0.5\right\} \cap \underset{i, \ell \varepsilon S, m=0,1, \ldots, r-1}{n}\left\{j: \mid P_{i, j}^{(m, n)} / P_{\ell, j}^{(m, n)}-\right. \\
\left.-P^{(m)}\left(T_{k} \mid X_{m}=i\right) / P^{(m)}\left(T_{k} \mid X_{m}=\right) \mid<\frac{1}{r}\right\} .
\end{aligned}
$$

But (6.3) implies

$$
\begin{aligned}
& \lim _{n^{\rightarrow \infty}}\left\{x _ { n } \varepsilon { } _ { 1 , \ell \varepsilon S , m = 0 , 1 , \ldots , r - 1 } ^ { n } \left\{j: \mid P_{i, j}^{(m, n)} / P_{\ell, j}^{(m, n)}-\right.\right. \\
& \left.-P^{(m)}\left(T_{k} \mid X_{m}=i\right) / P^{(m)}\left(T_{k} x_{m}=l\right) \mid<\frac{1}{r}\right\} \supseteq T_{k} \text { a.s. }
\end{aligned}
$$

for any $r$ and $k$. Take $r=2^{\nu}, \nu=1,2, \ldots$ For each $\nu$ one can find a number $\mathrm{m}(\nu)$ such that

$$
\begin{aligned}
& P^{(m)}\left(T_{k} \Delta \underset{n \geqslant m(\nu)}{U}\left\{x_{n} \varepsilon E_{n, k}^{(r)}\right\}\right) \leqslant 2^{-\nu}, k=1, \ldots, d \\
& P^{(m)}\left(T_{k} \Delta \underset{n \geqslant m(\nu)}{n}\left\{x_{n} \varepsilon E_{n, k}^{(r)}\right\}\right) \leqslant 2^{-\nu}, k=1, \ldots, d
\end{aligned}
$$

Define now $E_{n}^{(k)}=E_{n, k}^{(r)}$ for $m(\nu) \leqslant n<m(\nu+1)$ and consider the elementary set properties

$$
\left.\begin{array}{l}
\mathrm{A} \Delta(\mathrm{B} \cap \mathrm{C}) \\
\mathrm{A} \Delta(\mathrm{B} \cup \mathrm{C})
\end{array}\right\} \subseteq(\mathrm{A} \Delta \mathrm{B}) \cup(\mathrm{A} \Delta \mathrm{C})
$$


Using (6.4) yields

$$
\begin{aligned}
& P^{(m)}\left(T_{k} \Delta \underset{n \geqslant m(\nu)}{U}\left\{x_{n} \varepsilon E_{n}^{(k)}\right\}\right)=P^{(m)}\left(T_{k} \Delta \underset{u=\nu m(u) \leqslant n<m(u+1)}{U}\left(\underset{n}{U}\left\{E_{n, k}^{\left(2^{u}\right)}\right\}\right)\right. \\
& \leqslant \sum_{u=\nu}^{\infty} P^{(m)}\left(T_{k} \Delta \underset{n(u) \leqslant n<m(u+1)}{U}\left\{x_{n} \in E_{n, k}^{\left(2^{u}\right)}\right\}\right) \\
& \leqslant \sum_{u=v}^{\infty} P^{(m)}\left(T_{k} \Delta \underset{n \geqslant m(u)}{U}\left\{x_{n} \varepsilon E_{n, k}^{\left(2^{u}\right)}\right\}\right)+\sum_{u=}^{\infty} P^{(m)}\left(T_{k} \Delta \prod_{n \geqslant m(u)}\left\{x_{n} \varepsilon E_{n, k}^{\left(2^{u}\right)}\right\}\right) \\
& \leqslant 2^{-(\nu-2)} \text {. }
\end{aligned}
$$

Similarly, one can show

$$
\mathrm{P}^{(\mathrm{m})}\left(\mathrm{T}_{\mathrm{k}} \Delta \underset{\mathrm{n} \geqslant \mathrm{m}(\nu)}{n}\left\{\mathrm{X}_{\mathrm{n}} \in \mathrm{E}_{\mathrm{n}}^{(\mathrm{k})}\right\}\right) \leqslant 2^{-(\nu-1)}
$$

which boils down to

$$
\lim _{n \rightarrow \infty} P_{i, j}^{(t, n)} / P_{\ell, j}^{(t, n)}=P^{(m)}\left(T_{k} \mid X_{t}=i\right) / P^{(m)}\left(T_{k} \mid x_{t}=l\right)
$$

for $j \varepsilon E_{n}^{(k)}$ and $i, j \varepsilon E_{t}^{+}$. If we notice now that

$$
\alpha_{u}^{(k)}(m)=P^{(m)}\left(T_{k} \mid x_{m}=u\right)=\lim _{n \rightarrow \infty} P^{(m)}\left(\left\{x_{n} \varepsilon E_{n}^{(k)}\right\} \mid x_{m}=u\right)
$$

we get that (6.1) holds for states $i, j$ in $E_{t}^{+}$which depend on the $m$ that was chosen at the beginning of the proof. We sha1l nest show that (6.1) holds for every $t$ with the same sequences $\left\{\mathrm{E}_{\mathrm{n}}(\mathrm{k})\right\}$ constructed above for a particular $m$. Indeed, assume $m^{\prime} \neq m$. Then with our choice of $m E_{n}^{+} \supseteq E_{n}^{\prime^{+}}$ for $n>\max \left(m, m^{\prime}\right)$, and

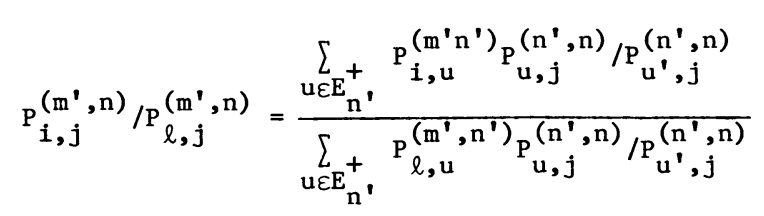

where $u^{\prime}$ is a state in $E_{n^{\prime}}^{+}$, with $P_{u^{\prime}, j}^{\left(m^{\prime}, n\right)}>0$. Since $\pi_{u}^{\left(n^{\prime}\right)} \pi_{u^{\prime}}^{\left(n^{\prime}\right)}>0$ the ratio $\mathrm{P}_{\mathrm{u}, \mathrm{j}^{\prime}}^{\left(\mathrm{n}^{\prime}, \mathrm{n}\right)} / \mathrm{P}_{\mathrm{u}^{\prime}, j}^{\left(\mathrm{n}^{\prime}, \mathrm{n}\right)}$ makes sense on the probability space attached to the chain $\left\{x_{n}: n \geqslant m\right\}$ and $(6.5)$ implies

$$
\lim _{n \rightarrow \infty} P_{u, j}^{\left(n^{\prime}, m\right)} / P_{u^{\prime}, j}^{\left(n^{\prime}, n\right)}=P^{(m)}\left(T_{k} \mid x_{n^{\prime}}=u\right) / P^{(m)}\left(T_{k} \mid x_{n^{\prime}}=u^{\prime}\right)
$$

where $j \varepsilon E_{n}^{(k)}$. Using this in (6.6) yields

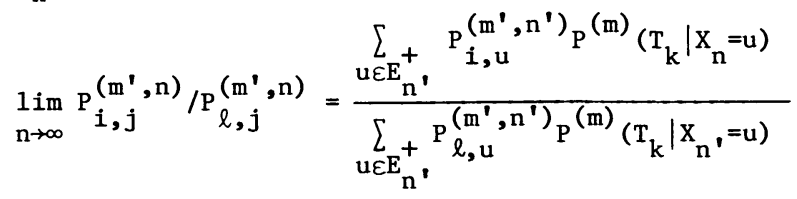

$$
\begin{aligned}
& =\frac{P^{(m)}\left(T_{k} \mid x_{m^{\prime}}=i\right)}{P^{(m)}\left(T_{k} \mid x_{m^{\prime}}=l\right)}
\end{aligned}
$$

which completes the proof of $(6.1)$.

To prove (6.2) notice that $\left\{\mathrm{X}_{\mathrm{n}} \varepsilon \mathrm{U}_{\mathrm{k}=1}^{\mathrm{d}} \mathrm{E}_{\mathrm{n}}^{(\mathrm{k})}\right.$ i.o. $\}=\Omega \mathrm{P}{ }^{(\mathrm{m})}$ a.s. which entails $\mathrm{P}^{(\mathrm{m})}\left(\mathrm{X}_{\mathrm{n}} \varepsilon \mathrm{E}_{\mathrm{n}}\right.$ i.o. $)=0$. This, in turn implies $\lim _{\mathrm{n} \rightarrow \infty} \mathrm{P}^{(\mathrm{m})}\left(\mathrm{X}_{\mathrm{n}} \varepsilon \mathrm{E}_{\mathrm{n}}\right)=0$ and proves $(6.2)$. 
The tail $\sigma$-field of a finite Markov chain was proven to be finite in Cohn [2]. Further, Senchenko [19] and Cohn [3] have independently shown by different methods that the number of atomic sets does not exceed the number of states. The proof of Theorem 6.1 given here was taken from Cohn [4]. Iosifescu [11] has studied the tail $\sigma$-field structure of continuous time Markov processes. Kingman [13] has given a geometrical representation of the transition matrices of a nonhomogeneous Markov chain from which the tail $\sigma$-field structure may be derived.

As far as the asymptotic behaviour of transition probabilities is concerned, it seems that the first result in the case of nonasymptotical independent chains was given by Blackwell [1] who derived the existence of limits for the reverse transition probabilities. However, Blackwell's paper does not refer to the tail $\sigma$-field notion. The results of this section on the asymptotics of $\left\{\mathrm{P}_{\mathrm{m}, \mathrm{n}}\right\}$ are derived in slightly different forms in Cohn [5] and [7]. 7. SOME CLASSES OF CHAINS CONSIDERED BY DOEBLIN.

In an important, but little known paper published in 1937, Doeblin [9] introduced a number of nonhomogeneous Markov chain models and gave without proofs several results concerning their asymptotic behaviour. One model was defined by the following

CONDITION $\left(D_{1}\right)$. There exists a strictly positive number $\delta$ such that for any fixed states $(i, j)$ either $p_{i, j}^{(n)} \geqslant \delta$ for all $n$ or $p_{i, j}^{(n)}=0$ for all $n$.

Doeblin asserted that in this case it is possible to decompose $S$ into disjoint 'final classes' $G_{0}, G_{1}, \ldots, G_{v}$, and each final class $G_{\alpha}, 1 \leqslant \alpha \leqslant v$ may be further decomposed into 'cyclical subclasses' $\left\{c_{i}(\alpha) ; i=1, \ldots, d(\alpha)\right\}$. These have the following asymptotic properties (according to Doeblin) as $n \rightarrow \infty$

(i) $\quad P_{i, j}^{(m, n)} \rightarrow 0$ for every $i \varepsilon S$ and $j \varepsilon G_{0}$;

(ii) $P_{i, j}^{(m, n)}=0$ for every $i \varepsilon G_{\alpha}$ and $j \notin G_{\alpha}$;

(iii) if $i, j \in G_{\alpha}$ with $i \varepsilon C_{\ell}(\alpha)$ and $j \varepsilon C_{\ell^{\prime}}(\alpha)$ then $P_{i, j}^{(m, n)}=0$ provided that $\mathrm{n}-\mathrm{m} \neq\left(\ell^{\prime}-l\right) \bmod \mathrm{d}(\alpha)$;

(iv) if $i, j \varepsilon G_{\alpha}$ with $i \varepsilon C_{\ell}(\alpha), j \varepsilon C_{\ell^{\prime}}(\alpha)$ then $P_{i, j}^{(m, n)}=P_{j}^{(n)}+\varepsilon_{i, j}^{(m, n)}$ provided that $n-m=\left(l^{\prime}-l\right) \bmod d(\alpha)$.

Here $\varepsilon_{i, j}^{(m, n)} \rightarrow 0$ exponentially as $n \rightarrow \infty$ for any $m, i$ and $j$ and the limit distribution $\left\{P^{(n)}\right\}$ satisfies $\sum_{j \in C_{\ell^{\prime}}(\alpha)} P_{j}^{(n)}=1$.

(v) for $i \varepsilon U_{\alpha=0}^{v} G_{\alpha}, j \varepsilon C_{\ell}(\alpha)$ and some $1 \leqslant \ell \leqslant d(\alpha), \alpha=1, \ldots, v$ $\mathrm{P}_{i, j}^{(m, n)}=P^{(m)}\left[i, \bar{C}_{n, l}{ }^{(\alpha)] P_{j}^{(n)}}+\varepsilon_{i, j}^{(m, n)}\right.$ where $P_{j}^{(n)}, \varepsilon_{i, j}^{(m, n)}$ are as in (iv) and $P^{(m)}\left[i, \bar{C}_{n, l}(\alpha)\right]$ is the limit as $r \rightarrow \infty$ of the probability, given $x_{m}=i$, that $x_{n+r d(\alpha)} \varepsilon C_{\ell}(\alpha)$.

Doeblin subsequently relaxed assumption (A) allowing positive $P_{i, j}^{(n)}$ to tend to 0 as $n \rightarrow \infty$. More precisely, he considered 
CONDITION $\left(\mathrm{D}_{2}\right)$. There exists a strictly positive number $\delta$ and some $N \geqslant 1$ such that for any fixed pair of states $(i, j)$ either $p_{i, j}^{(n)} \geqslant \delta$ for $n \geqslant N$ or $\lim _{n \rightarrow \infty} p_{i, j}^{(n)}=0$.

Two subclasses of chains satisfying Condition $\left(D_{2}\right)$ were further considered:

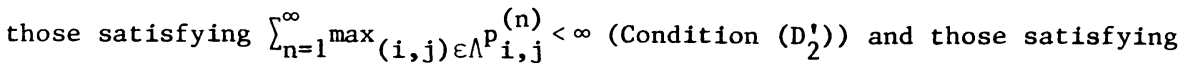
$\sum_{n=1}^{\infty} \max (i, j) \varepsilon \Lambda_{i, j}^{(n)}=\infty$ (Condition $\left(D_{2}^{\prime \prime}\right)$ ) where $\Lambda=\left\{(i, j): \lim _{n \rightarrow \infty} p_{i, j}^{(n)}=0\right\}$.

To study chains satisfying Condition $\left(\mathrm{D}_{2}\right)$ Doeblin proposed introducing an associated chain, derived from the initial one by taking 0 for the positive one-step transition probabilities tending to 0 . However, by so doing the transition matrices become nonstochastic, and it seems to us that Doeblin intended to add the transition probabilities replaced by 0 to the ones bounded away from 0 in the same row to preserve the stachasticity of the matrix. But there is considerable leeway in defining a matrix in this way and Doeblin's details are rather sketchy.

In the case $\left(D_{1}\right)$ it will be easily seen that the associated chain may be defined in any way described above, but in general we shall have to use some arguments based on the tail $\sigma$-field structure to justify the definition that we are going to adopt for an associated chain.

We proceed now to define an associated matrix. For the sake of definiteness we shall consider a matrix in which the entries of the initial matrix replaced by 0 are all added to the first entry in their row larger than $\delta$. More precisely, we say that $\mathrm{P}_{n}^{\prime}=\left(\mathrm{p}_{i, j}^{\prime}(\mathrm{n})\right.$ ) is an associated matrix of $\mathrm{P}_{n}$ if $\mathrm{p}_{i, j}^{\prime}(\mathrm{n})=0$ for $(i, j) \varepsilon \Lambda ; p_{i, j(i)}^{\prime(n)}=p_{i, j(i)}^{\prime(n)}+\sum_{j \in S_{i}} p_{i, j}^{(n)}, j(i)$ being the first entry in the ith row such that $(i, j) \notin \Lambda$ and $s_{i}=\{j:(i, j) \varepsilon \Lambda\}$, and $p_{i, j}^{\prime(n)}=p_{i, j}^{(n)}$ for the pairs $(i, j)$ such that $(i, j) \notin \Lambda$ and $j>j(i)$.

A Markov chain assuming the initial probability vector $\pi^{(\mathrm{m})}$ and the transition matrices $\left(P_{n}^{\prime}\right)_{n \geqslant m}$ will be said to be associated to $\left\{x_{n}: n \geqslant m\right\}$.

Denote by $\left\{E_{n}^{*}: n=0,1, \ldots\right\}$ a sequence of sets with the property $\lim \inf { }_{n \rightarrow \infty} \min \varepsilon_{i \varepsilon E_{n}^{*}} \pi_{i}^{(n)}>0$ and write $E_{n}^{* *}=S-E_{n}^{*}$. If $\left\{E_{n}^{* *}\right\}$ are present, then $\lim \inf _{n \rightarrow \infty} \max _{i \varepsilon E * *} \pi_{i}^{(n)}=0$.

LEMMA 7.1. Suppose that there exists a sequence of positive integers $m_{1}<n_{1}<m_{2}<n_{2}<\ldots$ such that $\sum_{u=1}^{\infty} P_{i, j}^{\left(m_{u}, n_{u}\right)}=\infty$ where $i \varepsilon E_{m_{u}}^{*}$ and $j \varepsilon s$. Then $\mathrm{P}^{(\mathrm{m})}\left(\mathrm{x}_{\mathrm{n}_{\mathrm{u}}}=\mathrm{j}\right.$ i.o. $)>0$.

PROOF. Using the argument employed in the proof of Theorem 3.3 we get that $P^{(m)}\left(A_{u} i . o_{.}\right)>0$ where $A_{u}=\left\{x_{m_{u}}=i, x_{m_{u}}=j\right\}, u=1,2, \ldots$ But $P^{(m)}\left(x_{n_{u}}=j\right.$ i.o. $) \geqslant P^{(m)}\left(A_{u}\right.$ i.o. $)>0$ as stated.

In what follows we shall consider a condition that contains $\left(D_{1}\right)$. We call this Condition $\left(D_{1}^{*}\right)$ :

(i) either the $\left\{E_{n}^{* *}\right\}$ are empty or $\lim _{n \rightarrow \infty} \pi_{i}^{(n)}=0$ for $i \varepsilon E_{n}^{* *}$, and 
(ii) the sequence $\left\{p_{i, j}^{(n)}: i \quad s, n=1,2, \ldots\right\}$ may contain ${ }^{\prime}$ 's but its positive values are bounded away from 0 , i.e. there exists $\delta>0$ such that inf ' $_{i, j, n}\left(p_{i, j}^{(n)}\right)>0$ where inf' means that the infinum is taken over the strictly positive matrices of the sequence.

Notice that $\left(D_{1}^{*}\right)(i i)$ holds under $\left(D_{1}\right)$. As far as $\left(D_{1}^{*}\right)(i)$ is concerned an arbitrary associated matrix $\mathrm{P}_{n}^{\prime}$, say $\mathrm{P}_{0}^{\prime}$, may be considered and taking into account only the position of its positive and null entries one may derive the periodicity and the cyclically moving subclasses of a homogeneous Markov chain assuming such type of transition probability matrix. Choosing $D$ to be a multiple of $\{d(\alpha), \alpha=1, \ldots, v\}$ we can easily conclude that $P_{m, n+N D}$ have all the positive and null entries in the same position as $\mathrm{P}_{1}^{\mathrm{ND}}$ and that $\left\{P \underset{i, j}{(n, n+N D)}: i, j \in C_{k}(\alpha), k=1, \ldots, d(\alpha)\right\}$ are all positive for $N$ sufficiently large, $\delta^{\mathrm{ND}}$ being a lower bound of these entries. Using this we can show that $C_{k}(\alpha) \cap E_{n}^{*}=C_{k}(\alpha)$, since for $j \varepsilon C_{k}(\alpha), \pi_{j}^{(n+N D)} \geqslant c \delta^{N D}$ with $c=\min { }_{i \varepsilon S} \pi_{i}^{(n)}$. Therefore $E_{n}^{*} \supseteq U_{\alpha=1}^{v} G_{\alpha}$ for $n$ sufficiently large. Further, it is easy to see that $\underset{n}{E *}=G_{0}$, and it will be seen that $G_{0}$ plays in this case the role of a 'transient' set.

THEOREM 7.1. Suppose that $\left(D_{1}^{*}\right)$ holds. Then

(i) there exists a sequence of disjoint events of $S$, say $E_{n}^{(1)}, \ldots, E_{n}^{(d)}, n=0,1, \ldots$ and a positive integer $N$ such that for any $m=0,1, \ldots$

$$
\bigcup_{n=N}^{\infty}\left\{X_{n} \varepsilon E_{n}^{(k)}\right\}=T_{k} \quad P^{(m)} \text { a.s. }
$$

for $k=1, \ldots, d$.

(ii) If $E_{n}=E-U_{k=1}^{d} E_{n}^{(k)}$ are present, then for $i \varepsilon S, m=0,1, \ldots$ and $j \varepsilon E_{n}$

$$
\lim _{n \rightarrow \infty} P_{i, j}^{(m, n)}=0 \text {. }
$$

For $i \in S, m=0,1, \ldots j \in E_{n}^{(k)}, n \geqslant N$

$$
P_{i, j}^{(m, n)}=\pi_{j}^{(n)} \frac{P_{n=N}^{(m)}\left(U_{n}^{\infty}\left\{E_{n}^{(k)}\right\} \mid x_{m}=i\right)}{P^{(m)}\left(\bigcup_{n=N}^{\infty}\left\{x_{n} \varepsilon E_{n}^{(k)}\right\}\right)}+o\left(\pi_{j}^{(n)}\right)
$$

For $i \in E_{m}^{(k)}, j \in E_{n}^{(k)}, m \geqslant N$

$$
P_{i, j}^{(m, n)}=\frac{\pi_{j}^{(n)}}{P^{(m)}\left(\bigcup_{n=N}^{\infty}\left\{x_{n} \varepsilon E_{n}^{(k)}\right\}\right)}+o\left(\pi_{j}^{(n)}\right)
$$

For $i \varepsilon E_{m}^{(k)}, j \notin E_{n}^{(k)}, m, n \geqslant N$

$$
\mathrm{P}_{i, j}^{(m, n)}=0
$$


PROOF. We shall first prove that for $n$ sufficiently large $\underset{n}{E_{n}^{* *}}=E_{n}$, where $P^{(m)}\left(X_{n} \in E_{n} i . o_{.}\right)=0$. Suppose that for an infinity of $n^{\prime} s p_{i, j}^{(n)} \geqslant \delta$ where $i \varepsilon E_{n}^{*}$ and $j \varepsilon E_{n+1}^{* *}$. Then $\pi_{j}^{(n+1)} \geqslant \delta \pi_{i}^{(n)}$. But 1 im inf ${ }_{n \rightarrow \infty} \pi_{i}^{(n)}>0$; this entails lim inf ${ }_{n \rightarrow \infty} \pi_{j}(n)>0$, which is impossible. Thus, $p_{i, j}^{(n)}=0$ whenever $i \varepsilon E_{n}^{*}$ and $j \in E_{n+1}^{* *}$ for $n$ sufficiently large. It follows that $P_{i, j}^{(m, n)}=0$ for $i \varepsilon E_{m}^{*}$ and $j \varepsilon \underset{n}{E *}$ and by Theorem 6.2 we get that $E_{n}^{(k)}{ }_{n}^{E_{n}^{* *}}$ must be empty for $n \geqslant N$ since otherwise $(6.2)$ would be invalidated. Therefore $E_{n}^{(k)} n E_{n}^{*}=E_{n}^{(k)}$ for $n$ sufficiently large and according to Lemma $7.1, p_{i, j}^{(n)} \geqslant \delta$ may happen only for finitely many $n$ with $i \varepsilon E_{n}^{(k)}$ and $j \in E_{n+1}^{\left(k^{\prime}\right)}$ with $k^{\prime} \neq k$. It follows that $\mathrm{P}^{(\mathrm{m})}\left(\left\{\mathrm{X}_{\mathrm{n}} \varepsilon \mathrm{E}_{\mathrm{n}}^{(\mathrm{k})}\right\} \mid\left\{\mathrm{X}_{\mathrm{n}-1} \varepsilon \mathrm{E}_{\mathrm{n}-1}^{(\mathrm{k})}\right\}\right)=1$ for $\mathrm{k}=1, \ldots, \mathrm{d}$ and $\mathrm{n} \geqslant \mathrm{N}$ which in conjunction with $\lim _{n \rightarrow \infty}\left\{x_{n} \varepsilon E_{k}^{(n)}\right\}=T_{k} \quad P^{(m)}$ a.s. yields $T_{k}=U_{n=N}^{\infty}\left\{x_{n} \varepsilon E_{n}^{(k)}\right\}$ and (7.1) is proved.

It is easy to see that Theorem 6.2 may now be applied to conclude (7.3) and (7.4). Finally, (7.2) follows from Lemma 7.1 , and (7.4) is a consequence of the proven part (i).

REMARK 7.1. For the chain satisfying Condition $\left(D_{1}\right)\left\{E_{n}^{(k)}\right\}$ are the subclasses $\left\{c_{k}(\alpha)\right\}$ in their cyclical order, i.e.

$$
\left\{\mathrm{E}_{\mathrm{n}}^{(\mathrm{k})}\right\}=\left\{\mathrm{c}_{\mathrm{u}+\mathrm{n}(\bmod \mathrm{d}(\alpha))}(\alpha)\right\}
$$

where $u=1, \ldots, d(\alpha), \alpha=1, \ldots, v$ and $k=1, \ldots, \sum_{\alpha=1}^{v} d(\alpha)$. Indeed, $\left.\mathrm{P}^{(\mathrm{m})}\left(\left\{\mathrm{x}_{\mathrm{n}} \varepsilon \mathrm{C}_{\mathrm{u}+\mathrm{n}(\bmod \mathrm{d}(\alpha))}(\alpha)\right\} \mid \mathrm{x}_{\mathrm{n}-1}=\mathrm{C}_{\mathrm{u}+\mathrm{n}-1(\bmod \mathrm{d}(\alpha))}(\alpha)\right\}\right)=1$ and $\lim _{\mathrm{n} \rightarrow \infty}\left\{\mathrm{x}_{\mathrm{n}} \varepsilon \mathrm{C}_{\mathrm{u}+\mathrm{n}(\bmod \mathrm{d}(\alpha))}(\alpha)\right\}=\Lambda_{\mathrm{u}}$ (say) $\mathrm{P}^{(\mathrm{m})}$ a.s. Besides, $\Lambda_{\mathrm{u}}$ belongs to $Y(m)$ and therefore it is either $\mathrm{P}^{(\mathrm{m})}$-atomic or a union of $\mathrm{P}^{(\mathrm{m})}$-atomic sets of $J^{(m)}$. Since $\left\{P_{i, j}^{(n, n+N D)} ; i, j \in C_{k}(\alpha), k=1, \ldots, d(\alpha)\right\}$ are positive for all $n$. If $\Lambda_{u}$ were not $P(m)$-atomic then there would exist two $P^{(m)}$-atomic subsets of $\Lambda_{u}, \Lambda_{u}^{\prime}$ and $\Lambda_{u}^{\prime \prime}$ and by Theorem 7.1 there would also exist two sequences of sets $\left\{E_{n}^{\prime}\right\}$ and $\left\{E_{n}^{\prime \prime}\right\}$ such that $U_{n \geqslant N}\left\{x_{n} \varepsilon E_{n}^{\prime}\right\}=\Lambda_{u}^{\prime} \quad p^{(m)}$ a.s. and $U_{n \geqslant N}\left\{X_{n} \varepsilon E_{n}^{\prime \prime}\right\}=\Lambda_{u}^{\prime \prime}$ $\mathrm{P}^{(\mathrm{m})}$ a.s., in which case (7.5) would be contradicted. This proves Doeblin's results stated before.

We next consider conditions which contain as particular cases Doeblin's Conditions $\left(D_{1}^{\prime}\right)$ and $\left(D_{2}^{\prime \prime}\right)$. First we consider

CONDITION (B). There exists $\delta>0$ such that if $\Lambda_{n}=\left\{(i, j): p_{i, j}^{(n)}<\delta\right\}$ then $\max _{(i, j) \varepsilon \Lambda_{n}} p_{i, j}^{(n)} \rightarrow 0$ as $n \rightarrow \infty$.

For chains satisfying Condition (B) we consider an associated chain in the same way as in the case $\left(D_{1}^{*}\right), i . e$. , we define the associated matrices $\left(P_{n}^{\prime}\right)$ in which the entries of the initial matrix replaced by 0 are all added to the first entry larger than $\delta$ in their row. In fact the whole definition of an associated matrix given before may be copies here word for word, the only difference being that $\Lambda$ is replaced by $\Lambda_{n}$, where $\Lambda_{n}=\left\{(i, j): p_{i, j}^{(n)}<\delta\right\}$; 
$S_{i}$ and $j(i)$ also depend on $n$ and should be denoted by $s_{i, n}$ and $j(i, n)$ respectively.

Let us next consider

CONDI'TION (C). There exist sequences of disjoint sets $\left\{F_{n}^{(\alpha)}: n=0,1, \ldots\right\}$ $\alpha=1, \ldots, d^{\prime}$ with $d^{\prime} \geqslant 2$ and a number $N$ such that $p_{i, j}^{(n)}=0$ for $i \varepsilon F_{n}^{(\alpha)}$ and $j \notin F_{n+1}^{(\alpha)}$ for $n \geqslant N, \alpha=1, \ldots, d^{\prime}$.

Theorem 7.1 shows that $\left(D_{1}^{*}\right)$ is a particular case of (C); the difference between these two conditions lies in that $\lim _{n \rightarrow \infty}\left\{x_{n} \varepsilon F_{\alpha}^{(n)}\right\}=U_{n=N}^{\infty}\left\{x_{n} \varepsilon F_{\alpha}^{(n)}\right\}$ $\mathrm{P}^{(\mathrm{m})}$ a.s. need not be an $\mathrm{P}^{(\mathrm{m})}$-atomic set of $J^{(\mathrm{m})}$, i.e. it may be a union of $p^{(m)}$-atomic sets of $J^{(m)}$.

We are now in the position to formulate the following two conditions.

CONDITION $\left(D_{2}^{*}\right)$. (i) (B) is satisfied with $\sum_{n=1}^{\infty} \max (i, j) \varepsilon \Lambda_{n} p_{i, j}^{(n)}<\infty$;

(ii) the associated matrices satisfy ( $\left.D_{1}^{*}\right)$.

CONDITION $\left(D_{2}^{* *}\right)$ ( $(i)$ (B) is satisfied with $\sum_{n=1}^{\infty} \max _{(i, j) \varepsilon \Lambda_{n} p_{i, j}^{(n)}=\infty}$;

(ii) the associated matrices satisfy (C).

THEOREM 7.2. If $\left(D_{2}^{*}\right)$ holds, then for $i \in S, m=0,1, \ldots$ and $j \in E_{n}^{\prime}$

$$
\lim _{n \rightarrow \infty} P_{i, j}^{(m, n)}=0
$$

and for $i \varepsilon S$ and $j \in E_{n}^{\prime(k)}, k=1, \ldots, d^{\prime}$

$$
P_{i, j}^{(m, n)}=\pi_{j}^{(n)} \frac{P^{(m)}\left(T_{k} \mid x_{m}=i\right)}{P^{(m)}\left(T_{k}\right)}+o\left(\pi_{j}^{(n)}\right) .
$$

PROOF. It is easy to see that if $A=\left\{\left(x_{n}, \ldots, X_{n+N}\right) \varepsilon B\right\}$ for $B \subset \underbrace{S \times \ldots \times S}_{N+1}$, and if $i \varepsilon\left\{j: \pi_{i}^{(m)} \pi_{j}^{(m)}>0\right\}$, then

$$
\left|P^{(m)}\left(A \mid X_{n}=i\right)-P^{\prime(m)}\left(A \mid X_{n}^{\prime}=i\right)\right| \leqslant \varepsilon_{n}
$$

where $\varepsilon_{n}=\sum_{k=n}^{\infty} \sum_{(i, j) \varepsilon \Lambda_{k}} p_{i, j}^{(k)}$, for $n>m$ and any $N>1$. The standard monotone class argument extends $(7.8)$ to any $A \in \mathcal{F}_{\mathrm{n}}$.

Suppose that we choose an associated chain $\left\{X_{n}^{\prime}: n \geqslant u\right\}$ such that $\pi_{i}^{(u)}>0$

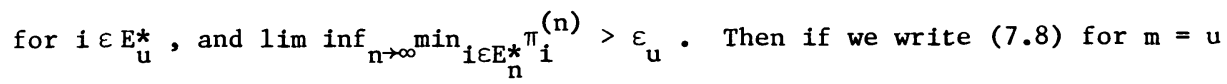
and $i \varepsilon\left\{j: \pi_{j}^{(u)}>0\right\}$, and take $A=\left\{x_{n}=j\right\}$ we get

$$
\left|P_{i, j}^{(u, n)}-P_{i, j}^{i(u, n)}\right| \leqslant \varepsilon_{u}
$$

But $\pi_{j}^{(n)}=\sum_{i \varepsilon S} \pi_{i}^{(u)} P_{i, j}^{(u, n)}, \pi_{j}^{(n)}=\sum_{i \varepsilon S} \pi_{i}^{(u)} P_{i, j}^{\prime(u, n)}$ and therefore

$$
\left|\pi_{j}^{(n)}-\pi_{j}^{(n)}\right| \leqslant \sum_{i \in S} \pi_{i}^{(u)}\left|P_{i, j}^{(u, n)}-P_{i, j}^{(u, n)}\right| \leqslant \varepsilon u
$$

On the other hand (7.9) implies 


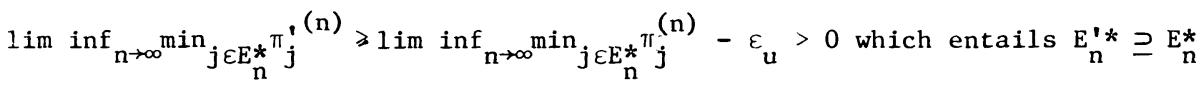
for $n$ sufficiently large. Take now $A=\left\{X_{n}, \varepsilon E_{n}^{\prime * *}\right\}$ in (7.8). Since

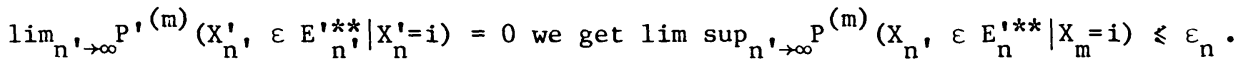
Further it is easy to see that $\left\{j: \pi_{j}^{\prime(n)}>0\right\} \subseteq\left\{j: \pi_{j}^{(n)}>0\right\}$ and since $\pi_{j}^{\prime(m)}>0$ for $j \in E_{m}^{*}$ and $m$ sufficiently large, we get $\lim \sup _{n \rightarrow \infty} P^{(m)}\left(\left\{X_{n} \varepsilon E_{n}^{\prime * *}\right\} \cap\left\{X_{m} \varepsilon E_{m}^{*}\right\}\right) \leqslant \varepsilon_{m}$. But the sequence $\left\{E_{n}^{*}\right\}$ has the property $\lim \sup _{n \rightarrow \infty} P^{(m)}\left(X_{n} \in E_{n}^{*}\right)=1$. This leads to $\lim _{n \rightarrow \infty} P^{(m)}\left(X_{n} \varepsilon E_{n}^{\prime * *}\right)=0$ and therefore $E_{n}^{*}=E_{n}^{* \prime}$ and $E_{n}^{\prime * *}=E_{n}^{* *}$ for $n$ sufficiently large.

Consider now a $\mathrm{P}^{(\mathrm{m})}$-atomic set of $\mathcal{J}^{(\mathrm{m})}$, say $\mathrm{T}_{\mathrm{k}}$. Then by Theorem 7.1 $\lim _{n \rightarrow \infty}\left\{X_{n} \varepsilon E_{n}^{(k)}\right\}=T_{k} P^{(m)}$ a.s. and (7.8) implies

$$
\left|P^{(m)}\left(\bigcup_{r=n}^{\infty}\left\{X_{r} \varepsilon E_{r}^{(k)}\right\} \mid X_{m}=i\right)-P^{\prime(m)}\left(\bigcup_{r=n}^{\infty}\left\{X_{r}^{\prime} \varepsilon E_{r}^{(k)}\right\} \mid X_{m}^{\prime}=i\right)\right| \leqslant \varepsilon_{m}
$$

and

$$
\left|P^{(m)}\left(\bigcap_{r=n}^{\infty}\left\{X_{r} \varepsilon E_{r}^{(k)}\right\} \mid X_{m}=i\right)-P^{\prime(m)}\left(\bigcap_{r=n}^{\infty}\left\{X_{r}^{\prime} \varepsilon E_{r}^{(k)}\right\} \mid X_{m}^{\prime}=i\right)\right| \leqslant \varepsilon_{m}
$$

Taking the limit over $\mathrm{n}$ in (7.11) and (7.12) and using the triangle inequality yields

$$
\left|P^{\prime(m)}\left(\lim _{n^{\prime} \rightarrow \infty} \sup _{n}\left\{X_{n}^{\prime} \varepsilon E_{n}^{(k)}\right\} \mid X_{m}^{\prime}=i\right)-P^{\prime(m)}\left(\lim _{n^{\prime} \rightarrow \infty} \inf \left\{X_{n}^{\prime} \varepsilon E_{n}^{(k)}\right\} \mid X_{m}^{\prime}=i\right)\right| \leqslant 2 \varepsilon_{n} .
$$

Multiplying (7.13) by $\pi_{i}^{(m)}$, assuming over $i$ and taking the limit over $m$ we get that $\lim _{n \rightarrow \infty}\left\{X_{n}^{\prime} \varepsilon E_{n}^{(k)}\right\}$ a.s. with respect to $P^{\prime(m)}$ exists, has positive probability and is either $P^{\prime(m)}$-atomic or a union of $P^{\prime(m)}$-atomic sets of $J^{\prime(m)}$. We may now interchange $P^{(m)}$ and $P^{\prime(m)}$ and get that

$$
\left|P^{(m)}\left(\lim \sup _{n \rightarrow \infty}\left\{X_{n}^{\prime} \varepsilon E_{n^{\prime}}^{\prime(k)}\right\} \mid X_{m}=i\right)-P^{(m)}\left(\lim _{n \rightarrow \infty} \inf _{n \rightarrow \infty}\left\{X_{n}^{\prime} \varepsilon E_{n^{\prime}}^{\prime(k)}\right\} \mid X_{m}=i\right)\right| \leqslant 2 \varepsilon_{m}
$$

Now because $J^{(m)}$ and $J^{(m)}$ are finite, we conclude that their atomic sets are in a one-to-one correspondence. Therefore $d=d^{\prime}$ and

$$
\begin{aligned}
& P^{(m)}\left(\lim _{n \rightarrow \infty}\left\{X_{n} \varepsilon E_{n}^{(k)}\right\} \Delta \lim _{n \rightarrow \infty}\left\{X_{n} \varepsilon E_{n}^{\prime\left(k^{\prime}\right)}\right\}\right)=0 \\
& P^{\prime(m)}\left(1 i_{n \rightarrow \infty}\left\{X_{n}^{\prime} \varepsilon E_{n}^{(k)}\right\} \Delta \lim _{n \rightarrow \infty}\left\{X_{n}^{\prime} \varepsilon E_{n}^{\prime(k)}\right\}\right)=0
\end{aligned}
$$

for $k, k^{\prime} \varepsilon\{1, \ldots, d\}$. Since we have seen in the proof of Theorem 7.1 that $E_{n}^{\prime(k)} \cap E_{n}^{\prime *}=E_{n}^{\prime *}$ for $n$ large enough it follows that $E_{n}^{\prime\left(k^{\prime}\right)} \subseteq E_{n}^{(k)}$ for $k$ sufficiently large. Now it is easy to see that $P^{(m)}\left(\left\{X_{n} \varepsilon E_{n}^{\prime} i .0.\right\}\right)=0$ and complete the proof by an already familiar reasoning.

REMARK 7.2. The sets $E_{n}^{\prime(k)}$ corresponding to the associated chain $\left\{X_{n}^{\prime}: n \geqslant m\right\}$ are in general smaller than the sets $\left\{\mathrm{E}_{\mathrm{n}}^{(\mathrm{k})}\right.$ \} for which Theorem 7.1 guarantees the same convergence property (7.7). It is therefore possible that there exists states $i_{u} \varepsilon E_{n}^{\prime}$ with $i_{u} \varepsilon E_{n}^{(k)}$ for some $k$ and $n$. However such states 
have the property $P^{(m)}\left(X_{n}=i_{u}\right.$ i.o. $)=0$. The usefulness of using $\left\{E_{n}^{(k)}\right\}$ instead of $\left\{\mathrm{E}_{\mathrm{n}}^{(\mathrm{k})}\right\}$ lies in the fact that the former are more easily obtainable. For example in case $\left(D_{2}^{\prime}\right)$ we have seen that such sets may be identified by means of an arbitrary one-step transition probability matrix.

We turn now to the case ( $\left.D_{2}^{*}\right)$ which is considerably more complicated than the ones considered so far. We shall first need the following

LEMMA 7.2. Suppose that the sequence of $\operatorname{sets}\left\{A_{n}\right\}$ is such that for $\mathrm{m}_{1}<\mathrm{n}_{1}<\mathrm{m}_{2}<\mathrm{n}_{2}<\ldots$ and a sequence $\left\{i_{k}\right\}$ with $i_{k} \in \mathrm{m}_{\mathrm{m}_{k}}^{*}, \mathrm{k}=1,2, \ldots$ and some $\mathrm{N} \geqslant 1$

$$
\sum_{k=1}^{\infty} \min _{i A_{n_{k}} P_{i_{k}, j}\left(m_{k}, n_{k}\right)}=\infty
$$

and $p_{i, j}^{(n)}=0$ for $i \varepsilon A_{n}$ and $j \varepsilon A_{n+1}$ with $n \geqslant N$. Then

(i) $\quad P^{(m)}\left(X_{n_{k}}=j_{k}\right.$ i.o. $)>0$ for $j_{k} \in A_{n_{k}}, k=1,2, \ldots$

(ii) $\lim _{n \rightarrow \infty} P_{i, j}^{(m, n)} / \pi_{j}^{(n)}=\frac{P^{(m)}\left(\bigcup_{n=N}^{\infty}\left\{x_{n} \varepsilon A_{n}\right\} \mid x_{m}=i\right)}{P^{(m)}\left(\bigcup_{n=N}^{\infty}\left\{x_{n} \varepsilon A_{n}\right\}\right)}$

for $i \varepsilon S, m=0,1, \ldots, j \in A_{n}, n=1,2, \ldots$

PROOF. (i) Let us consider the subchain $x_{m_{1}}, x_{n_{1}}, x_{m_{2}}, x_{n_{2}}, \ldots$ We shall show that $\lim _{k \rightarrow \infty}\left\{x_{n_{k}} \varepsilon A_{n_{k}}\right\}$ a.s. is a $P^{(m)}$-atomic set of $\mathcal{Y}_{1}(m)$, where $\mathcal{Y}^{\prime}(m)$ is the tail $\sigma-f$ ield of $\left\{x_{m_{1}}, x_{n_{1}}, \ldots s\right.$. Since $p_{i, j}^{(n)}=0$ for $i \varepsilon A_{n}$ and $j \varepsilon A_{n+1}$ with $n \geqslant N$, we get $U_{n=N}^{\infty}\left\{x_{n} \varepsilon A_{n}\right\}=1 i m_{k \rightarrow \infty}\left\{x_{n_{k}} \varepsilon A_{n_{k}}\right\} p^{(m)}$ a.s. If $1 i m_{k \rightarrow \infty}\left\{x_{n_{k}} \varepsilon A_{n_{k}}\right\}$ is not a $P^{(m)}$-atomic set of $\mathcal{J}^{(m)}$ then it must be a union of atomic sets of $J^{(m)}$ In the latter case, by Proposition 2.1, there must exist sequences $\left\{A_{n}^{(1)}, \ldots, A_{n^{\prime}}^{(v)}\right\}$ with $n^{\prime} \varepsilon\left\{m_{1}, n_{1}, m_{2}, n_{2}, \ldots\right\}$ such that $\lim _{n^{\prime} \rightarrow \infty}\left\{x_{n^{\prime}}, \varepsilon A_{n^{\prime}}^{(r)}\right\}=T^{(r)}$ (say) $\mathrm{P}^{(\mathrm{m})}$ a.s. for $\mathrm{r}=1, \ldots, \mathrm{v}$. Further, since $i_{k} \in \mathrm{E}_{\mathrm{m}_{\mathrm{k}}}^{*}$ there must be a number M such that $i_{k} \in U_{r=1}^{v} A_{m_{k}}^{(r)}$ for $k \geqslant M$. But $\left\{A_{n^{\prime}}^{(1)}, \ldots, A_{n^{\prime}}^{(v)}\right\}$ are disjoint for all $n$ and therefore there exist the sets $\Lambda_{1}, \ldots, \Lambda_{v}$ with $\Lambda_{r}=\left\{m_{k}: i_{k} \varepsilon A_{n_{m}}^{(r)}\right\}$, $r=1, \ldots, v$ and $U_{r=1}^{v} \Lambda_{r} \supseteq\left\{m_{k}: k \geqslant M\right\}$. Take $j_{k} \notin A_{n_{k}}^{(r)}$ for $m_{k} \varepsilon \Lambda_{r}$. Since $\sum_{k=M}^{\infty} P_{i_{k}, j_{k}}^{\left(m_{k}, n_{k}\right)} \leqslant \sum_{r=1}^{v} \sum_{m_{k}} \varepsilon \Lambda_{k} P_{i_{k}, j_{k}}^{\left(m_{k}, n_{k}\right)}$ if we apply Lemma 7.1 we get that

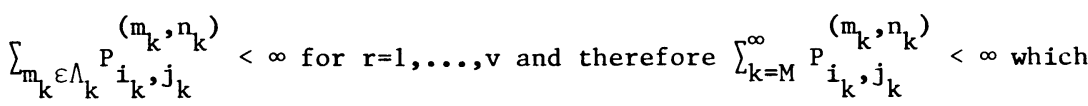
contradicts (7.15). Hence $\lim _{k \rightarrow \infty}\left\{x_{n_{k}} \varepsilon A_{n_{k}}\right\}$ a.s. is a $P^{(m)}$-atomic set of $\mathcal{G}^{\cdot(m)}$. Further $\lim _{k \rightarrow \infty}\left\{\mathrm{X}_{n_{k}} \in A_{n_{k}}\right\}=T^{\prime}$ a.s. is also a $P^{(m)}$-atomic set of $J^{(m)}$, since 
otherwise $f^{(\mathrm{m})}$ would contain at least two $\mathrm{P}^{(\mathrm{m})}$-atomic sets, $\mathrm{T}_{1}$ and $\mathrm{T}_{2}$. If $\left\{\mathrm{E}_{\mathrm{n}}^{(1)}\right\}$ and $\left\{\mathrm{E}_{\mathrm{n}}^{(2)}\right\}$ are some sequences corresponding to $\mathrm{T}_{1}$ and $\mathrm{T}_{2}$ such that $\lim _{n \rightarrow \infty}\left\{x_{n} \varepsilon E_{n}^{(1)}\right\}=T_{1} P^{(m)}$ a.s. and $\lim _{n \rightarrow \infty}\left\{x_{n} \varepsilon E_{n}^{(1)}\right\}=T_{1} P^{(m)}$ a.s. and $\lim _{n \rightarrow \infty}\left\{x_{n} \varepsilon E_{n}^{(2)}\right\}=T_{2} P^{(m)}$ a.s., we also get $\lim _{k \rightarrow \infty}\left\{x_{n_{k}} \varepsilon E_{n_{k}}^{(1)}\right\}=T_{1} P^{(m)}$ a.s. and $\lim _{k \rightarrow \infty}\left\{x_{n_{k}} \varepsilon E_{n_{k}}^{(2)}\right\}=T_{2} P^{(m)}$ a.s. contradicting the atomicity of $T^{\prime}$ with respect to $J^{(m)}$.

Suppose now that $j_{k} \in E_{n_{k}}^{*}$ for an infinity of $k^{\prime} s$. Then $\left\{x_{n_{k}}=j_{k}\right.$ i.o. $\}$ has positive $P^{(m)}$ probability. But $j_{k} \varepsilon A_{n_{k}}$ and as seen before $\lim _{k \rightarrow \infty}\left\{x_{n_{k}} \varepsilon A_{n_{k}}\right\}=T_{u}$ $\mathrm{p}^{(\mathrm{m})}$ a.s., which leads to $\left\{\mathrm{x}_{\mathrm{n}_{\mathrm{k}}}=\mathrm{j}_{\mathrm{k}}\right.$ i.o. $\}=\mathrm{T}_{\mathrm{u}} \mathrm{P}^{(\mathrm{m})}$ a.s. If $\mathrm{j}_{\mathrm{k}} \varepsilon \mathrm{E}_{\mathrm{n}_{\mathrm{k}} * *}$ for $\mathrm{k}$ large we get (i) by applying Lemma 7.1. Indeed, $i_{k} \varepsilon \mathrm{E}_{\mathrm{m}_{k}}^{*} \cap \mathrm{A}_{\mathrm{m}_{\mathrm{k}}}$ for $k$ sufficiently large and because $\lim _{k \rightarrow \infty}\left\{\mathrm{x}_{\mathrm{m}_{\mathrm{k}}} \varepsilon \mathrm{A}_{\mathrm{m}_{\mathrm{k}}}\right\}=\lim _{\mathrm{k} \rightarrow \infty}\left\{\mathrm{x}_{\mathrm{m}_{\mathrm{k}}} \varepsilon \mathrm{E}_{\mathrm{m}_{\mathrm{k}}}^{(\mathrm{u})}\right\}=\mathrm{T}_{\mathrm{u}}$ $p^{(m)}$ a.s. we get that $i_{k} \in E_{m_{k}}^{*} \cap E_{m_{k}}^{(u)}$ for $k$ sufficiently large and (i) follows.

To prove (ii) we shall first prove it for a subsequence $\left\{n_{k}^{\prime}: k=1,2, \ldots\right\}$ i.e. we shall show that $A_{n_{k}^{\prime}} \cap E_{n_{k}^{\prime}}^{(u)}=A_{n_{k}^{\prime}}$ for $k$ sufficiently large. Notice that $A_{n_{k}} \cap E_{n_{k}}$ is not empty for an infinity of $k^{\prime} s$. Indeed, by (7.15) and Lemma 7.1 it is impossible for this intersection to be nonempty for all $k$ sufficiently large, since then (i) would imply the positivity of $P^{(m)}\left(x_{n_{k}} \varepsilon A_{n_{k}} \cap E_{n_{k}}\right.$ i.o.) contradicting $\mathrm{P}^{(\mathrm{m})}\left(\mathrm{X}_{\mathrm{n}_{\mathrm{k}}} \varepsilon \mathrm{E}_{\mathrm{n}_{\mathrm{k}}}\right.$ i.o. $)=0$. Therefore there must exist a subsequence of $\left\{n_{k}\right\}$, say $\left\{n_{k}^{\prime}\right\}$, such that $A_{n_{k}^{\prime}} \cap E_{n_{k}}^{(u)}=A_{n_{k}^{\prime}}$ for $k=1,2, \ldots$ We shall further show that using the existence of such a subsequence $\left\{n_{k}^{\prime}\right\}$ we deduce (ii).

We first prove (ii) for $m \geqslant N$ and $i \in A_{m}$. Write $\gamma_{m, i}=P^{(m)}\left(T_{u} \mid x_{m}=i\right) / P(m)\left(T_{u}\right)$ and take $j \in A_{n}$ to get

$$
\begin{aligned}
& \left|\frac{P_{i, j}^{(m, n)}}{\pi_{j}^{(n)}}-\gamma_{m, i}\right|=\left|\frac{\sum_{l=1}^{s} P_{i, l}^{\left(m, n_{k}^{\prime}\right)} P_{\ell, j}^{\left(n_{k}^{\prime}, n\right)}}{\sum_{l=1}^{s} \pi_{l}^{\left(n_{k}^{\prime}\right)\left(n_{k}^{\prime}, n\right)}-\gamma_{m, i}}\right|
\end{aligned}
$$

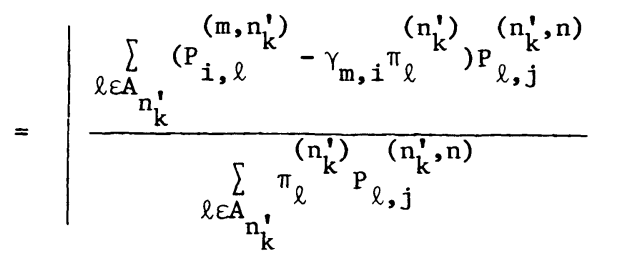




$$
\begin{aligned}
& =\left|\sum_{r \in A_{n_{k}^{\prime}}^{\prime}} \frac{\left(P_{i, r}{ }^{\left(m, n_{k}^{\prime}\right)}-\gamma_{m, i} \pi_{r}^{\left(n_{k}^{\prime}\right)}\right) P_{r, j}^{\left(n_{k}^{\prime}, n\right)}}{\sum_{l \varepsilon A_{n_{k}^{\prime}}} \pi_{l}^{\left(n_{k}^{\prime}\right){ }_{P}\left(n_{k}^{\prime}, n\right)}}\right| \\
& =\sum_{r \in A_{n_{k}^{\prime}}^{\prime}}\left|\frac{P_{i, r}^{\left(m, n_{k}^{\prime}\right)}-\gamma_{m, i_{r}} \pi_{k}^{\left(n_{k}^{\prime}\right)}}{\pi_{r}\left(n_{k}^{\prime}\right)}\right|
\end{aligned}
$$

where the prime in the last two sums indicates that the sum is restricted to the values $r$ such that $\mathrm{P}_{r, j}^{\left(n_{k}^{\prime}, n\right)}>0$. Now if $n \rightarrow \infty$ we may take $k \rightarrow \infty$ and Theorem 6.1 implies that the last sum in (7.16) goes to 0 as $k \rightarrow \infty$.

We prove now (ii) for arbitrary $m$ and $i$. Consider the conditional probabilities

$$
P_{i, j}^{*(m, r)}=P^{(m)}\left(x_{m^{\prime}+1} \notin A_{m^{\prime}+1}, \ldots, x_{r-1} \notin A_{r-1}, x_{r}=j \mid x_{m}=i\right)
$$

for $j \varepsilon A_{\ell}, \ell>N, m^{\prime}=\max (n-1, m)$ and $r>m^{\prime}+1$, and

$$
P_{i, j}^{*(m, m+1)}=P_{i, j}^{(m, m+1)}
$$

Since $\left\{x_{n} \varepsilon A_{n}\right\} \subseteq\left\{x_{n+1} \varepsilon A_{n+1}\right\}$ for $n \geqslant N$, a slight modification of a standard reasoning from the theory of homogenoeus chains yields

$$
\mathrm{P}_{i, j}^{(\mathrm{m}, \mathrm{n})}=\sum_{\ell=\mathrm{m}^{i}+1}^{\mathrm{n}-1} \sum_{k \in A_{\ell}} \mathrm{P}_{i, k}^{*(m, l)} \mathrm{P}_{\mathrm{k}, \mathrm{j}}^{(l, \mathrm{n})}+\mathrm{P}_{i, j}^{*(\mathrm{~m}, \mathrm{n})}
$$

We recall now that for $\ell \geqslant N$ and $k \varepsilon A_{\ell}$ we have already shown that $\lim _{n \rightarrow \infty} \frac{P_{k, j}^{(l, n)}}{\pi_{j}^{(n)}}=\gamma_{\ell, k}$ where $\gamma_{\ell, k}=\frac{1}{P^{(m)}\left(T_{u}\right)}$. It follows that for an arbitrary $\mathrm{N}^{\prime}$ with $\mathrm{N}^{\prime}>\mathrm{m}^{\prime}+1$

$$
\lim _{n \rightarrow \infty} \frac{\sum_{\ell^{\prime} m^{\prime}}^{N^{\prime}} \sum_{k \varepsilon A_{\ell}} P_{i, k}^{*(m, l)} P_{k, j}^{(l, n)}}{\pi_{j}^{(n)}}=\frac{P^{(m)}(\underbrace{N^{\prime}}_{\ell=m^{\prime}}\left\{x_{\ell} \varepsilon A_{\ell}\right\} \mid x_{m}=i)}{P^{(m)}\left(T_{u^{\prime}}\right)}
$$

Because $N^{\prime}$ was arbitrarily chosen (7.19) and (7.20) together imply

$$
\lim \inf \left(\frac{P_{i, j}^{(m, n)}}{\pi_{j}^{(n)}}-\frac{P^{(m)}\left(T_{u} \mid x_{m}=i\right)}{P^{(m)}\left(T_{u}\right)}\right) \geqslant 0
$$

for any $m$ and $i$.

Further

$$
\sum_{i \varepsilon S} \pi_{i}^{(m)} \frac{P_{i, j}^{(m, n)}}{\pi_{j}^{(n)}}=\sum_{i \varepsilon S} \pi_{i}^{(m)} \frac{P^{(m)}\left(T_{u} \mid x_{m}=i\right)}{P^{(m)}\left(T_{u}\right)}=1
$$

and (7.21) yields 


$$
\lim _{n \rightarrow \infty} \frac{P_{i, j}^{(m, n)}}{\pi_{j}^{(n)}}=\frac{P^{(m)}\left(T_{u} \mid x_{m}=i\right)}{P^{(m)}\left(T_{u}\right)}
$$

completing the proof.

THEOREM 7.3. Suppose that $\left(D_{2}^{*}\right)$ holds and let $\Omega_{1}, \ldots, \Omega_{\mathrm{d}}$ be a partition of $\left\{1, \ldots, \mathrm{d}^{\prime}\right\}$ and $\mathrm{E}_{\mathrm{n}}^{(\mathrm{k})}=U_{\alpha \varepsilon \Omega_{\mathrm{k}}} \mathrm{F}_{\mathrm{n}}^{(\alpha)}, \mathrm{k}=1, \ldots, \mathrm{d}$. If for any $\mathrm{k} \varepsilon\{1, \ldots, \mathrm{d}\}$ there exists a sequence of positive integers $m_{1}<n_{1}<m_{2}<n_{2}<\ldots$ and a sequence of states $i_{m_{1}}^{(k)}, i_{m_{2}}^{(k)}, \ldots$ such that $i_{m_{u}}^{(k)} \varepsilon \underbrace{*}_{m_{u}}$ for $u$ sufficiently large,

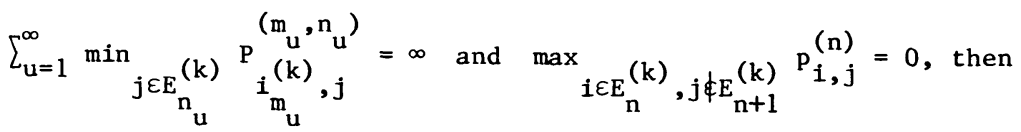

(i) for any $i_{n} \varepsilon E_{n}^{(k)}, P^{(m)}\left(x_{n}=i_{n} i .0.\right)>0, k=1, \ldots, d$;

(ii) for $i \varepsilon S, m=0,1, \ldots$ and $j \in E_{n}^{(k)}, n=1,2, \ldots$

$$
P_{i, j}^{(m, n)}=\pi_{j}^{(n)} \frac{P^{(m)}\left(T_{k} \mid x_{m}=i\right)}{P^{(m)}\left(T_{k}\right)}+o\left(\pi_{j}^{(n)}\right)
$$

where $T_{k}=\lim _{n \rightarrow \infty}\left\{x_{n} \varepsilon E_{n}^{(k)}\right\} \quad P^{(m)}$ a.s., $k=1, \ldots, d$;

(iii) for any $i \varepsilon s, m=0,1, \ldots$ and $j \varepsilon E_{n}=s-U_{k=1}^{d} E_{n}^{(k)}, n=1,2, \ldots$

$$
\lim _{n \rightarrow \infty} P_{i, j}^{(m, n)}=0
$$

(iv) if $\Omega_{k}$ contains more than one element and if $F_{n}^{(\alpha)} \subset E_{k}^{(n)}$ then the average sojourn time spent in the sequence of $\operatorname{sets}\left\{F_{n}^{(\alpha)}, F_{n+1}^{(\alpha)}, \ldots\right\}$ given that $x_{n}=i$ with $i \in F_{n}^{(\alpha)}$ goes to $\infty$ as $n \rightarrow \infty$, but $P^{(m)}\left(\left\{x_{n} \varepsilon F_{n}^{(\alpha)} u 1 t.\right\}\right)=0$.

PROOF. It is easy to see that (i) and (ii) follow from Lemma 7.2, whereas (iii) is implied by Theorem 6.2. It remains to prove (iv). Let us denote by $E\left(Y_{\alpha}^{(n)} \mid X_{n}=i\right)$ the average sojourn time referred to in the statement of the Theorem. By definition, $Y_{\alpha}^{(n)}=m$ on the set $\left\{X_{n} \varepsilon F_{n}^{(\alpha)}\right\} \cap \ldots \cap\left\{X_{n+m+1} \varepsilon F_{n+m-1}^{(\alpha)}\right\}$ $n\left\{x_{n+m} \notin F F_{n+m}^{(\alpha)}\right\}$ for $m=1,2, \ldots$ Because $\Omega_{k}$ contains more than one element, and by (i) $P^{(m)}\left(X_{n}=i_{n} i .0_{.}\right)>0$ for any sequence $\left\{i_{n}\right\}$ with $i_{n} \varepsilon E_{n}^{(k)}$, we conclude that $\mathrm{P}^{(\mathrm{m})}\left(\mathrm{Y}_{\alpha}^{(\mathrm{n})}<\infty\right)=1$. Further, it is easy to see that since $\sum_{j \in F_{n+k+1}^{(\alpha)}} P_{i, j}^{\prime(n+k)}=1$ for $i \in F_{n+k}^{(\alpha)}$ and any $k \geqslant 1$, we get $\sum_{j \in F_{n+m-1}^{(\alpha)}} P_{i, j}^{(n, n+m-1)}=1$ for $i \in F_{n}^{(\alpha)}$ and $m \geqslant 2$. Taking into account that $\left|p_{i, j}^{(n)}-p_{i, j}^{(n)}\right| \rightarrow 0$ as $n \rightarrow \infty$ for $a 11 i, j \in S$ we get

$$
\lim _{n \rightarrow \infty} P^{(n)}\left(\left\{x_{n} \varepsilon F_{n}^{(\alpha)}\right\} \cap \ldots \cap\left\{x_{n+m-1} \varepsilon F_{n+m-1}^{(\alpha)}\right\} \mid x_{n}=i\right)=1
$$

which implies that $\lim _{n \rightarrow \infty} E\left(Y_{\alpha}^{(n)} \mid X_{n}=i\right)=\infty$ for $i \varepsilon F_{n}^{(\alpha)}$ and $\alpha \varepsilon \Omega_{k}$. Finally, since $P^{(m)}\left(X_{n} \in F_{n}^{(\alpha)}\right.$ i.o. $)=P^{(m)}\left(X_{n} \in\left(E_{n}^{(k)}-F_{n}^{(\alpha)}\right)\right.$ i.o. $)=P^{(m)}\left(T_{k}\right)>0$ we get 
that $\mathrm{P}^{(\mathrm{m})}\left(\mathrm{X}_{\mathrm{n}} \in \mathrm{F}_{\mathrm{n}}^{(\alpha)} \mathrm{u} 1 \mathrm{t}_{.}\right)=0$ and the proof is complete.

As a corollary to Theorem 7.3 we shall give a result that describes the asymptotic behaviour of a chain that satisfies Condition $\left(D_{2}^{* *}\right)$.

COROLLARY 7.1. Suppose that $\left(D_{2}^{* *}\right)$ holds and let $\Omega_{1}, \Omega_{2}, \ldots, \Omega_{d}$ be a partition of $\{(\ell, \alpha), \ell=1, \ldots, v ; \alpha=1, \ldots, d(\alpha)\}$. Let $E_{k}^{(n)}=U_{(\ell, \alpha) \varepsilon \Gamma_{k}} C_{\ell}(\alpha)$, $k=1, \ldots, d$ and denote $\varepsilon_{(\ell, \alpha) ;\left(\ell^{\prime}, \alpha^{\prime}\right)}^{(n)}=\max _{i \varepsilon C_{\ell}(\alpha), j \in C_{\ell^{\prime}}(\alpha)} p_{i, j}^{(n)}$. Assume that $\sum_{n=1}^{\infty} \min (n)(l, \alpha) ;\left(l^{\prime}, \alpha^{\prime}\right)=\infty$ where the minimum is taken over a11 $\left\{(\ell, \alpha) ;\left(\ell^{\prime}, \alpha^{\prime}\right) \varepsilon \Omega_{k} \times \Omega_{k}, k=1, \ldots, d\right\}$, and that $\varepsilon_{(l, \alpha):\left(\ell^{\prime}, \alpha^{\prime}\right)}^{(n)}=0$ for $(l, \alpha) \varepsilon \Omega_{k}$ and $\left(\ell^{\prime}, \alpha^{\prime}\right) \notin \Omega_{k}$, Then the statement of Theorem 7.3 holds.

We shall omit the proof of this result, which may be carried out by arguments already used in this paper.

We notice that in the case of Condition $\left(D_{2}^{\prime \prime}\right)$ Doeblin's statement is wrong. However, examining Doeblin's formulae makes it clear that he felt that unlike the previous situations, the limit of the conditional probability that the chain will circulate through the cyclical subclasses of a fixed class may not exist here. The analogy to the homogeneous case seems to break down for the chains satisfying $\left(D_{2}^{\prime \prime}\right)$ since several atomic sets of the tail $\sigma$-field of the associated chain may be lumped into one atomic set of the tail $\sigma$-field of the original chain.

Theorem 7.3(iv) generalizes a result stated by Doeblin about chains satisfying Condition $\left(D_{2}^{\prime \prime}\right)$. It is hard to see how Doeblin could have reached his conclusions in this respect, given the knowledge available at the time his paper was written.

The results of this section, in slightly different form, were given in Cohn [7].

\section{WEAK ERGODICITY.}

One of the main concerns of the theory of finite stochastic matrices has been to characterize sequences of matrices satisfying the so-called 'weak ergodicity' condition, i.e.

$$
\lim _{n \rightarrow \infty}\left(P_{i, j}^{(m, n)}-P_{l, j}^{(m, n)}\right)=0
$$

for any $i, j, l$ and $m$. This condition has been introduced by Kolmogorov [14] and most papers on nonhomogeneous chains are related to it. Doeblin [9] has found necessary and sufficient conditions for (8.1) and Hajnal [10] has derived similar conditions unaware of Doeblin's results. We shall first give a result that relates weak ergodicity to the structure of the tail $\sigma$-field.

THEOREM 8.1. The following conditions are equivalent

(i) weak ergodicity;

(ii) any Markov chain $\left\{x_{n}: n \geqslant m\right\}$ with transition probability $\left(P_{n}\right)_{n \geqslant m}$ and arbitrary initial distribution $\pi^{(m)}$ has a $P^{(m)}$-trivial $\sigma$-field $\mathcal{J}^{(\mathrm{m})}$.

PROOF. Since $S$ is finite we may assume, if necessary after relabelling the states at successive times $n=0,1, \ldots$, that there is a positive state $j \in S$. 
Then Theorem 3.2 and Remark 3.1 imply that

$$
\begin{aligned}
& \lim _{n^{\prime} \rightarrow \infty} P_{i, j}^{\left(m, n^{\prime}\right)} / P_{\ell, j}^{\left(m, n^{\prime}\right)} \\
& n^{\prime} \varepsilon \Gamma_{k}
\end{aligned}
$$

If (8.1) holds then $\mathrm{P}^{(\mathrm{m})}\left(\mathrm{T}_{\mathrm{k}} \mid \mathrm{X}_{\mathrm{m}}=\mathrm{i}\right) / \mathrm{P}^{(\mathrm{m})}\left(\mathrm{T}_{\mathrm{k}} \mid \mathrm{X}_{\mathrm{m}}=\ell\right)=1$ necessarily follows by (8.2). However, if $\mathcal{J}^{(\mathrm{m})}$ is not $\mathrm{P}^{(\mathrm{m})}$-trivial this is not possible as by the martingale convergence theorem $\mathrm{P}^{(\mathrm{m})}\left(\mathrm{T}_{\mathrm{k}} \mid \mathrm{X}_{\mathrm{n}}=\mathrm{i}\right)$ must have values close to 0 for some $i$ in view of $\lim _{n \rightarrow \infty} P^{(m)}\left(T_{k} \mid x_{n}\right)=1_{T_{k}} P^{(m)}$ a.s. Thus $\mathcal{f}^{(m)}$ is $\mathrm{P}^{(\mathrm{m})}$-trivial. Suppose now that $\mathrm{J}^{(\mathrm{m})}$ is $\mathrm{P}^{(\mathrm{m})}$-trivial. Then by Theorem 6.2 we know that

$$
\lim _{n \rightarrow \infty} P_{i, j}^{(m, n)} / P_{\ell, j}^{(m, n)}=1
$$

for $i \notin E_{n}$. But $\lim _{n \rightarrow \infty} P^{(m)}\left(X_{n} \varepsilon E_{n}\right)=0$ for all $m$ and (8.1) follows

THEOREM 8.2. Let $\left(P_{n}\right)$ be a sequence of finite stochastic matrices. The following two conditions are equivalent:

(i) weak ergodicity;

(ii) there exists a sequence of sets $\left\{E_{n}^{(1)}\right\}$ such that for $i, l \in S$ and $m \varepsilon N$

$$
\lim _{\mathrm{n} \rightarrow \infty} \mathrm{P}_{i, j}^{(\mathrm{m}, \mathrm{n})} / \mathrm{P}_{\ell, j}^{(m, n)}=1
$$

for $j \in E_{n}^{(1)}$, and for any $i \in S$ and $m=0,1, \ldots$

$$
\lim _{n \rightarrow \infty} \sum_{j \in E_{n}^{(1)}} P_{i, j}^{(m, n)}=1
$$

This result is a consequence of Theorems 6.2 and 8.1 .

A classical type of results in the theory of nonhomogeneous Markov chains establishes weak ergodicity in terms of some coefficients attached to a stochastic matrix. A historical account of such coefficients, that goes back to Doeblin, may be found in Seneta [20]. Kingman [13] has proven a general result of this kind. Usually, the proof is carried out by some inequalities relating the coefficients of the product of two matrices to the coefficients of the matrices themselves. For example, Hajnal [10] considered the following coefficient attached to a matrix $P$ with entries $p_{\alpha, \beta}, 1 \leqslant \alpha \leqslant s, 1 \leqslant \beta \leqslant s$

$$
\{\mathrm{P}\}=\min _{\alpha, \alpha^{\prime}} \sum_{\beta=1}^{\mathrm{s}} \min \left(\mathrm{p}_{\alpha, \beta}, \mathrm{p}_{\alpha^{\prime}, \beta}\right)
$$

We show next that such results are immediate consequences of the results given in this paper by proving the following theorem due to Hajnal [10].

THEOREM 8.3. A sequence of stochastic matrices $\left(P_{n}\right)$ is weakly ergodic if there exists an increasing sequence of positive integers $n_{1}, n_{2}, \ldots$ such that $\sum_{j}\left\{P_{n_{j}, n_{n+1}}\right\}$ diverges.

PROOF. According to Theorem 8.1, $\left(\mathrm{P}_{n}\right)$ is not weakly ergodic if the tail $\sigma-f i e l d g^{(m)}$ is not $P^{(m)}$-trivial and thus assumes at least two $P^{(m)}$-atomic sets. According to Lemma 7.1 we must have 
$\sum_{j=1}^{\infty} P_{\alpha, \beta}^{\left(n_{j}, n_{j+1}\right)}<\infty$ whenever $\alpha \in E_{n_{j}}^{(k)} \cap E_{n}^{*}$ and $\beta \varepsilon E_{n_{j+1}}^{\left(k^{\prime}\right)}$ with $k \neq k^{\prime}$ which makes $\sum_{j}\left\{P_{n_{j}, n_{j+1}}\right\}$ convergent for any sequence $n_{1}<n_{2}<\ldots$, and finishes the proof.

There is an important result for bounded positive matrices known in the demographic 1iterature as the Coale-Lopez theorem (see Seneta [21]). The result was given in a somewhat more general form in Seneta [21] and its proof seems rather laborious. The specialization of the Coale-Lopez theorem to the case of stochastic matrices reveals a strong asymptotic independence property. We shall state such a property under a less restrictive assumption on the stochastic matrices.

THEOREM 8.4. Let $\left(P_{n}\right)$ be a weakly ergodic sequence of stochastic matrices such that $\lim \inf _{n \rightarrow \infty} \max _{i \in s} P_{i, j}^{(0, n)}>0$ for any $j \in S$. Then for all $i, l \in S$

$$
\lim _{n \rightarrow \infty} P_{i, j}^{(m, n)} / P_{\ell, j}^{(m, n)}=1
$$

The proof follows easily from Theorem 3.2 and Remark 3.1 in view of the fact that $\left\{\mathrm{E}_{\mathrm{n}}\right\}$ are empty.

The Lopez theorem imposes the condition $P_{i, j}^{\left(n, n+r_{0}\right)} \geqslant \delta>0$ for a certain $r_{0}$ and $\delta$ and any $\mathrm{n}$. This clearly implies weak ergodicity, since as seen in the course of the proof of Theorem 8.3, the failure of weak ergodicity prevents $\left(n, n+r_{0}\right)$

$\mathrm{P}_{i, j}$ from being bounded away from 0 for $a 11 i, j$ and $n$.

The results of this section were derived in Cohn [5].

\section{REFERENCES}

1. BLACKWELL, D. Finite non-homogeneous Markov chains, Ann. Math. 46, 594-599, 1945.

2. CoHN, H. On the tail $\sigma$-algebra inhomogeneous Markov chains, Ann. Math. Statist. 41, 2175-2176, 1970.

3. COHN, H. On the tail events of a Markov chain, Z. Wahrscheinlich. 29, 65-72, 1974 .

4. COHN, H. A ratio 1 imit theorem for the finite nonhomogeneous Markov chains, Israe1 J. Math. 19, 329-334, 1974.

5. COHN, H. Finite non-homogeneous Markov chains: Asymptotic behaviour, Adv. App1. Probab. 8, 502-516, 1976.

6. COHN, H. Countable non-homogeneous Markov chains: Asymptotic behaviour, Adv. App1. Probab. 9, 542-552, 1977.

7. COHN, H. On a paper by Doeblin on non-homogeneous Markov chains, Adv. App1. Probab. 13, 388-401, 1981.

8. COHN, H. On a class of non-homogeneous Markov chains, Math. Proc. Camb. Phil. Soc. 92, 527-534, 1982.

9. DOEBLIN, W. Le cas discontinu des probabilités en chaîne, Pub. Fac. Sci. Univ. Massryk (Brno) 236, 1937.

10. HAJNAL, J. Weak ergodicity in non-homogeneous Markov chains, Proc. Camb。 Phil. Soc. 54, 233-246, 1958.

11. IOSIFESCU, M. On finite tail o-algebras, Z. Wahrscheinlich. 24, 159-166, 1982 .

12. KEMENY, J.G., SNELL, L。J., and KNAPP, A.W. Denumerable Markov Chains, Springer, New York, 1976. 
13. KINGMAN, J.F.C. Geometrical aspects of the theory of non-homogeneous Markov chains, Math. Proc. Camb. Phil. Soc. 77, 171-185, 1975.

14. KOLMOGOROV, A.N. Uber die analytischen Methoden in der Wahrscheinlichkeitsrechnung, Math. Ann. 104, 415-418, 1931.

15. MAKSIMOV, V.M. Convergence of non-homogeneous biostochastic Markov chains, Theor. Probab. App1. 15, 604-618, 1970.

16. MUKHERJEA, A. Limit theorems: Stochastic matrices, erdogic Markov chains, and measures on semigroups. Probabilistic Analysis and Related Topics, Vol. 2, 143-203, Academic Press.

17. MUKHERJEA, A. A new result on the convergence of non-homogeneous stochastic chains, Trans. Amer. Math. Soc. 262, 505-520, 1980.

18. MUKHERJEA, A. and CHAUDHURI, R. Convergence of non-homogeneous stochastic chains II, Math. Proc。Camb. Phil. Soc. 90, 167-182, 1981.

19. SENCHENKO, D.V. The final $\sigma$-algebra of an inhomogeneous Markov chain with finite number of states, Math. Notes 12, 610-613, 1972.

20. SENETA, E. On the historical development of the theory of finite nonhomogeneous Markov chains, Proc. Camb. Phil. Soc. 74, 507-513, 1973.

21. SENETA, E. Non-negative Matrices, George Allen and Unwin Ltd., London, 1973.

22. WINKLER, G. The number of phases in inhomogeneous Markov fields with finite state spaces on $\mathrm{N}$ and $\mathrm{Z}$ and their behaviour at infinity, Math. Nachr. 104, 101-117, 1981 . 


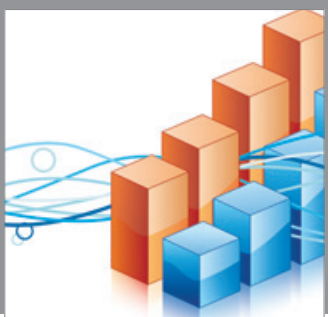

Advances in

Operations Research

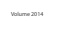

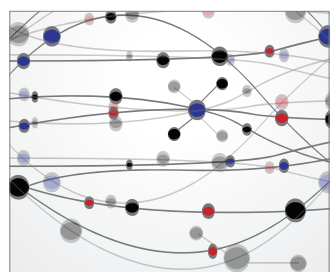

\section{The Scientific} World Journal
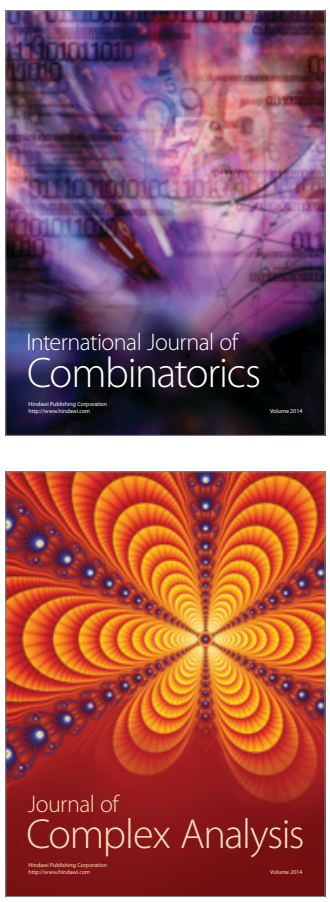

International Journal of

Mathematics and

Mathematical

Sciences
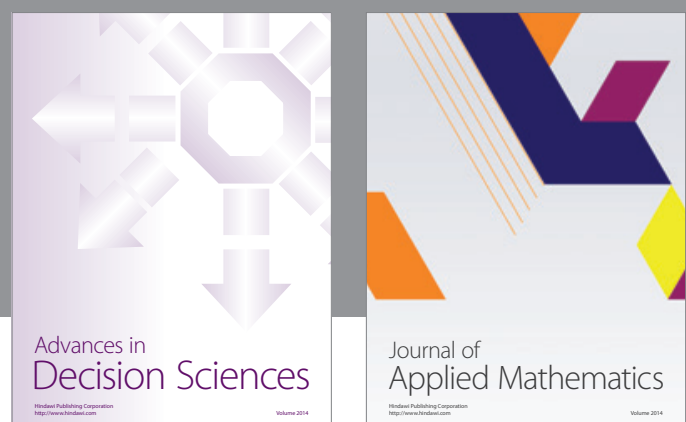

Journal of

Applied Mathematics
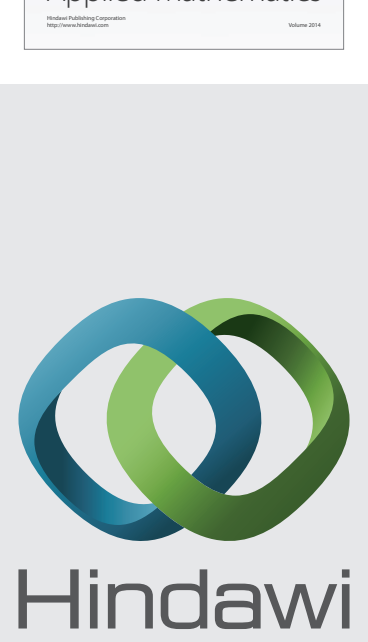

Submit your manuscripts at http://www.hindawi.com
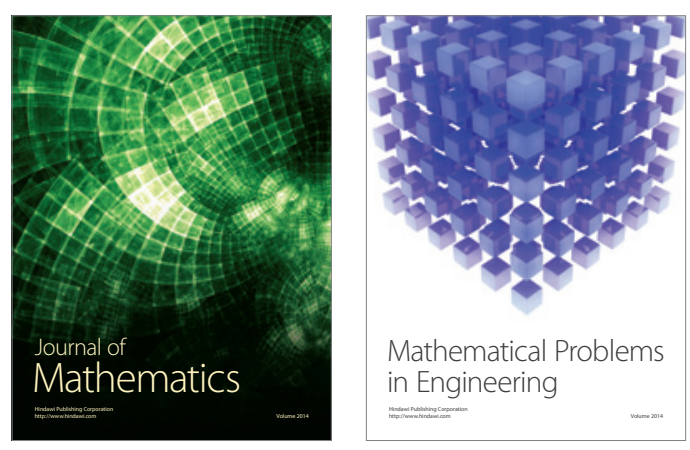

Mathematical Problems in Engineering
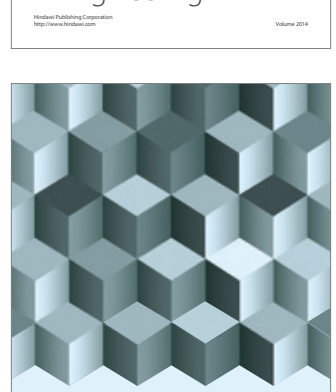

Journal of

Function Spaces
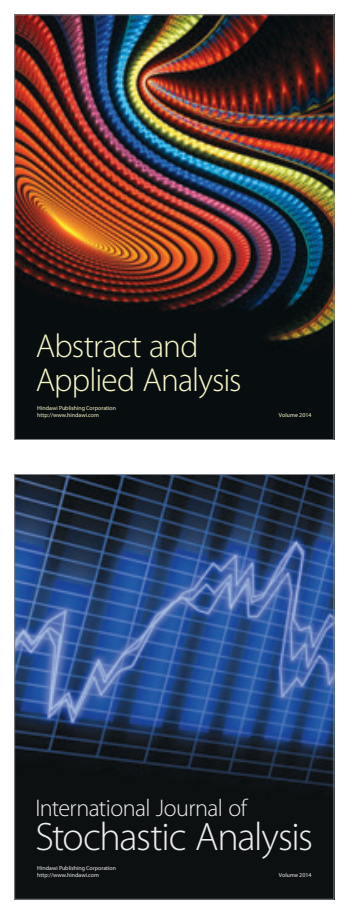

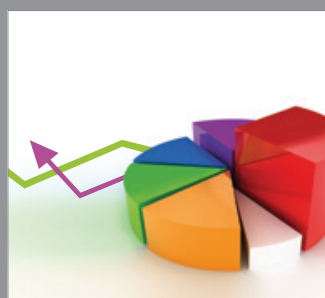

ournal of

Probability and Statistics

Promensencen
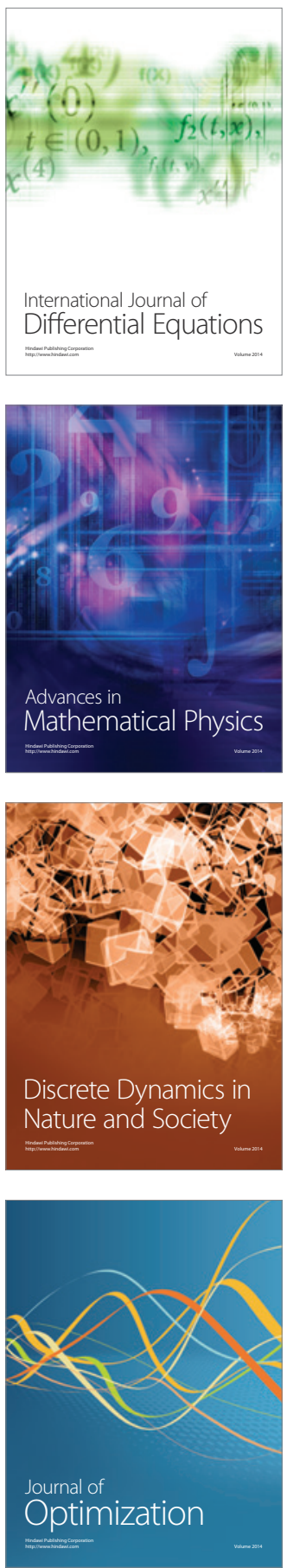